\title{
On the structure of nonarchimedean exponential fields I*
}

\author{
Salma Kuhlmann
}

31. 8.1993

\begin{abstract}
Given an ordered field $K$, we compute the natural valuation and skeleton of the ordered multiplicative group $\left(K^{>0}, \cdot, 1,<\right)$ in terms of those of the ordered additive group $(K,+, 0,<)$. We use this computation to provide necessary and sufficient conditions on the value group $v(K)$ and residue field $\bar{K}$, for the $L_{\infty}$-equivalence of the above mentioned groups. We then apply the results to exponential fields, and describe $v(K)$ in that case. Finally, if $K$ is countable or a power series field, we derive necessary and sufficient conditions on $v(K)$ and $\bar{K}$ for $K$ to be exponential. In the countable case, we get a structure theorem for $v(K)$.
\end{abstract}

\section{Introduction}

Given an ordered field $K$, let $v$ be its natural valuation, with value group $G=$ $v\left(K^{\times}\right)$and residue field $\bar{K}$. Let $v_{G}$ be the natural valuation of $G$. Classical results on the model theory of valued fields have successfully shown how to analyze (elementary) properties of a valued field through those of its value group and residue field. One of the aims of this paper is to do something analoguous for exponential fields.

What axioms should we require for an exponential? A hint to answer this question is to think about the best known exponential field, namely the ordered field of the reals endowed with the exponential function $\exp (x)$. In view of the recent progress in the model theory of this structure (cf. [W], [D-M-M], [RE], [M-W]) and the proof of the model completeness of its elementary theory $\operatorname{Th}((\mathbb{R}, \exp (x)))$, it is desirable to give concrete descriptions of the models. So understanding the algebraic structure of exponential fields is important, since it will provide methods for the construction of examples as we shall see later.

In this article, we shall first consider the simplest case: an exponential field here is a pair $(K, f)$ where $K$ is an ordered field and $f$ is an order preserving group

*1) This paper represents some results of the author's doctoral thesis.

2) This paper was written while the author was supported by a research grant from the university of Heidelberg. 
isomorphism from the additive group of the field onto the multiplicative group of positive elements. Of course, $f$ will also have to induce some extra structure on $G$ and on $\bar{K}$, if we want to do any reasonable analysis of $(K, f)$. So, for instance, we would like $f$ to induce a canonical map $\bar{f}$ on $\bar{K}$ (given by $\bar{f}(\bar{a})=\overline{f(a)}$ ). This is equivalent to demanding that

$$
v(f(1)-1)=0
$$

which in turn is equivalent to the assumption that $f$ maps the valuation ideal $I_{v}$ onto the group of 1 -units $1+I_{v}$ (cf. Lemma 3.20). So we will always require (1) to be satisfied in an exponential field (although (1) is not an elementary sentence in the language of ordered fields, it may be replaced by an elementary, but stronger, one - cf. section 3.3). Exponentials satisfying further axioms such as the growth and the Taylor axioms, will constitute the subject of the subsequent paper [K-K1].

We have seen above how to obtain $(\bar{K}, \bar{f})$ for a given $(K, f)$. It is more complicated to do the same with the value group, and it requires first an understanding of the structure of the multiplicative group $\left(K^{>0}, \cdot, 1,<\right)$ of positive elements, where $K$ is an arbitrary ordered field, not necessarily endowed with an exponential. This anlysis is done in section 3.2, and it turns out to be very fruitful indeed in its applications to exponential fields. The necessary preliminaries are given in chapter 2. The crucial notion appearing there is that of the skeleton $S(G)$ of an ordered Abelian group $G$. The important result of section 3.2 is Theorem 3.19 which computes the natural valuation and the skeleton of $\left(K^{>0}, \cdot, 1,<\right)$ in terms of those of $(K,+, 0,<)$ and $G$.

In section 3.3 we then apply the results of section 3.2 to exponential fields, where $\left(K^{>0}, \cdot, 1,<\right)$ and $(K,+, 0,<)$ are isomorphic through an exponential $f$ and thus have the same skeleton (since this is an invariant for ordered Abelian groups). Using the computation of Theorem 3.19, we are able to show that the existence of an exponential $f$ puts a very restrictive condition on the value group of $K$ (which we will say to be an exponential group — cf. our definition preceding to Theorem 3.23). In particular, $f$ induces in a canonical way an isomorphism of chains

$$
\varphi_{f}: G^{<0} \rightarrow \Gamma^{-}
$$

where $\Gamma^{-}=v_{G}(G \backslash\{0\})$ denotes the value set of $G \backslash\{0\}$. We are now able to formulate the following more concrete problems to which this paper and the subsequent paper [K-K1] try to provide an answer:

1) Study the structure and (elementary) properties of $(K, f)$ through those of $(\bar{K}, \bar{f})$ and $\left(G, \varphi_{f}\right)$.

2) Assume that $G$ is an exponential group and $\bar{K}$ admits an exponential $\bar{f}$ : when is it possible to lift it to an exponential $f$ of $K$ ?

3) Given an archimedean exponential field $(E, e)$ satisfying good properties (e.g. $e$ is continuous, differentiable and equal to its own derivative), give a construction of a nonarchimedean exponential field $(K, f)$ satisfying the same good properties and such that $\bar{K}=E$ and $\bar{f}=e$.

In the case where $K$ is a nonarchimedean countable field, root closed for positive elements, we get a surprisingly simple result. Indeed, not only we are able to give an answer to the above problems, but moreover we get quite a strong structure 
theorem for those fields (cf. Theorem 3.33). In fact, given an exponential $\bar{f}$ on $\bar{K}$, such a field $K$ admits an exponential $f$ lifting $\bar{f}$ if and only if $G$ is isomorphic to the lexicographic sum of copies of the additive ordered group $(\bar{K},+, 0,<)$, taken over the rationals. Theorem 3.33 will also provide an answer to the third problem, as is shown in $[\mathrm{K}-\mathrm{K} 1]$.

The interest of this theorem is that it reduces the construction of nonarchimedean countable exponential fields to that of archimedean ones. Indeed, given a countable exponentially closed subfield $E$ of $\mathbb{R}$, just take a countable valued field having $E$ as its residue field and $\coprod_{\mathbb{Q}} E$ as its value group ( $\amalg$ denotes lexicographic sums).

A main ingredient of the proof of Theorem 3.33 is a result of $\mathrm{R}$. Brown on countable valued vector spaces: any two countable valued vector spaces with isomorphic skeletons are isomorphic. Now let us remark that in order to compute the skeleton of $\left(K^{>0}, \cdot, 1,<\right)$, we prove that for every ordered field, we have

$$
S\left(I_{v},+, 0,<\right) \simeq S\left(1+I_{v}, \cdot, 1,<\right),
$$

in other words, the valuation ideal (seen as an additive ordered group) and the multiplicative group of 1-units always have the same skeleton (cf. Corollary 3.15). Hence, by Brown's Theorem, we know that if $K$ is any countable ordered field, root closed for positive elements (which is equivalent to the assertion that $\left(K^{>0}, \cdot, 1,<\right)$ be divisible), then it admits a right exponential, i.e. an isomorphism

$$
f: I_{v} \longrightarrow 1+I_{v}
$$

of ordered groups. Here, the word "right" is suggested by the lexicographic decompositions $(K,+, 0,<)=\mathbf{A} \amalg \mathbf{A}^{\prime} \amalg I_{v}$ and $\left(K^{>0}, \cdot, 1,<\right)=\mathbf{B} \amalg \mathbf{B}^{\prime} \amalg 1+I_{v}$ given in Lemma 3.4 and Theorem 3.8. Now if in addition $G \simeq \coprod_{\mathbb{Q}} \bar{K}$, then we can find an isomorphism $h$ between the "left" parts $\mathbf{A}$ and $\mathbf{B}$ of these groups. The "middle" parts $\mathbf{A}^{\prime}$ and $\mathbf{B}^{\prime}$ are taken care of by $\bar{f}$. The "lexicographic product" of $h, \bar{f}$ and $f$ will then be the required exponential.

This principle of decomposing those groups lexicographically and then showing that the respective parts are isomorphic in order to "put an exponential together" will also be used several times in [K-K1], to obtain a strengthening of Theorem 3.33. The left parts are always taken care of by our conditions on the value group $G$, and the middle parts by conditions on the residue field $\bar{K}$. A final remark concerning question 1) above: an extensive model theory for the value groups of exponential fields is now given in [KF2] and [KF3]. Also the relation between the exponential field and its residue field in the o-minimal case is by now studied in [D-L]. At this point, I would like to thank F.-V. Kuhlmann for many useful discussions. I would also like to thank Arne Ledet and N.L. Alling for a hint concerning these notes, and all other participants of our seminar for their interest and patience.

\section{$2 \quad$ Valued and ordered modules}

\subsection{Generalities about valued modules}

All modules considered in this section are left $R$-modules, for a fixed ring $R$. The definitions and results of this section also cover the case of valued Abelian groups 
since they may be considered as $\mathbb{Z}$-modules. If $\left\{M_{i} ; i \in I\right\}$ is a family of modules, then $\bigoplus_{i \in I} M_{i}$ will denote the direct sum.

In the sequel, let $M$ be a module and $\Gamma$ a chain (i.e., a totally ordered set) with last element $\infty$. A surjective map

$$
v: M \longrightarrow \Gamma
$$

is a valuation on $M$ (and $(M, v)$ is a valued module) if for all $x, y \in M$ and $r \in R$, the following holds:

(i) $v(x)=\infty$ if and only if $x=0$,

(ii) $v(r x)=v(x)$ if $r \neq 0$,

(iii) $v(x-y) \geq \min \{v(x), v(y)\}$.

Axiom (ii) says that the scalar multiplication by nonzero elements preserves the value. So we might speak of a "valued module with value preserving scalar multiplication" in contrast to modules equipped with a map $v$ which only satisfies axioms (i) and (iii). There are also important applications of the latter, more general notion of a valued module. Both notions will be considered in subsequent papers (see $[\mathrm{KF} 1])$. In this paper, we will only need valued modules satisfying axiom (ii), so we will suppress the specification "with value preserving scalar multiplication". Note that axiom (ii) together with axiom (i) implies that $M$ is torsion free.

The following is a consequence of the above axioms:

$$
v(x) \neq v(y) \Longrightarrow v(x+y)=\min \{v(x), v(y)\} .
$$

We abbreviate $\Gamma \backslash\{\infty\}$ by $\Gamma^{-}$and call it the rank of $M$. The restriction of $v$ to a submodule of $M$ is a valuation on that submodule.

Let $\left(M_{1}, \Gamma_{1}, v_{1}\right),\left(M_{2}, \Gamma_{2}, v_{2}\right)$ be two valued modules and

$$
h: M_{1} \longrightarrow M_{2}
$$

an isomorphism of $R$-modules. We will say that $h$ preserves the valuation (or that $h$ is an isomorphism of valued modules) if there exists an isomorphism of chains

$$
\varphi: \Gamma_{1} \longrightarrow \Gamma_{2}
$$

such that for all $x \in M_{1}$,

$$
\varphi\left(v_{1}(x)\right)=v_{2}(h(x)) .
$$

We will say that $\left(M_{1}, \Gamma_{1}, v_{1}\right)$ and $\left(M_{2}, \Gamma_{2}, v_{2}\right)$ are isomorphic as valued modules if such an isomorphism $h$ exists. Similarly, $h$ is an embedding of valued modules if $h$ is an isomorphism of valued modules of $M_{1}$ onto a submodule of $M_{2}$. We will omit "of valued modules" and "as valued modules" if the context is clear. Two valuations $v_{1}$ and $v_{2}$ on $M$ are called equivalent, if the identity map on $M$ is an isomorphism between the valued modules $\left(M, v_{1}(M), v_{1}\right)$ and $\left(M, v_{2}(M), v_{2}\right)$.

Remark 2.1 The isomorphism $h: M_{1} \longrightarrow M_{2}$ preserves the valuation if and only if the map

$$
h_{\mathrm{rk}}: \Gamma_{1} \longrightarrow \Gamma_{2}
$$

given by

$$
h_{\mathrm{rk}}\left(v_{1}(x)\right)=v_{2}(h(x))
$$

is well defined and an isomorphism of chains. 
By an ordered system of modules we mean a pair

$$
[\Gamma,\{B(\gamma) ; \gamma \in \Gamma\}]
$$

where $\{B(\gamma) ; \gamma \in \Gamma\}$ is a family of modules indexed by $\Gamma$ such that $B(\gamma)=0$ if and only if $\gamma=\infty$.

Let $S_{i}=\left[\Gamma_{i},\left\{B_{i}(\gamma) ; \gamma \in \Gamma_{i}\right\}\right]$ be an ordered system of modules, for $i=1,2$. We will say that $S_{1}$ and $S_{2}$ are isomorphic if there exists an isomorphism

$$
\varphi: \Gamma_{1} \longrightarrow \Gamma_{2}
$$

of chains, and for every $\gamma \in \Gamma_{1}$, an isomorphism

$$
\varphi_{\gamma}: B_{1}(\gamma) \longrightarrow B_{2}(\varphi(\gamma))
$$

of modules. Then we will call $\left[\varphi,\left\{\varphi_{\gamma} ; \gamma \in \Gamma_{1}\right\}\right]$ an isomorphism and write

$$
\left[\varphi,\left\{\varphi_{\gamma} ; \gamma \in \Gamma_{1}\right\}\right]: S_{1} \simeq S_{2}
$$

Let $\gamma \in \Gamma$ and put

$$
\begin{aligned}
& M^{\gamma}=\{x \in M ; v(x) \geq \gamma\} \\
& M_{\gamma}=\{x \in M ; v(x)>\gamma\} .
\end{aligned}
$$

Then $M^{\gamma}, M_{\gamma}$ are submodules (for $\gamma \neq \infty$ ) satisfying $M_{\gamma} \subset M^{\gamma} \subset M$. We put

$$
B(M, \gamma)=M^{\gamma} / M_{\gamma}, B(M, \infty)=0
$$

We will say that $B(M, \gamma)$ is the component corresponding to $\gamma$. The skeleton of $(M, \Gamma, v)$, denoted by $S(M)$, is the ordered system $[\Gamma,\{B(M, \gamma) ; \gamma \in \Gamma\}]$. We will write $B(\gamma)$ instead of $B(M, \gamma)$ if the context is clear, and in what follows, $B_{i}(\gamma)$ instead of $B\left(M_{i}, \gamma\right)$, for $i=1,2$.

For every $\gamma \in \Gamma$, the coefficient map corresponding to $\gamma$ is the canonical homomorphism

$$
\pi^{M}(\gamma,-): M^{\gamma} \longrightarrow B(\gamma) \text { defined by } \pi^{M}(\gamma, x)=x+M_{\gamma},
$$

and we write $\pi(\gamma,-)$ instead of $\pi^{M}(\gamma,-)$ if the context is clear.

The following lemma shows that an isomorphism of two valued modules induces an isomorphism of their skeletons:

Lemma 2.2 Suppose that $h: M_{1} \longrightarrow M_{2}$ is an isomorphism of valued modules. Then for all $\gamma \in \Gamma_{1}$, the map

$$
h_{\gamma}: B_{1}(\gamma) \longrightarrow B_{2}\left(h_{\mathrm{rk}}(\gamma)\right)
$$

defined by

$$
\pi^{M_{1}}(\gamma, x) \mapsto \pi^{M_{2}}\left(h_{\mathrm{rk}}(\gamma), h(x)\right)
$$

is an isomorphism of modules. Hence,

$$
\left[h_{\mathrm{rk}},\left\{h_{\gamma} ; \gamma \in \Gamma_{1}\right\}\right]: S\left(M_{1}\right) \simeq S\left(M_{2}\right) .
$$


Let $[\Gamma,\{B(\gamma) ; \gamma \in \Gamma\}]$ an ordered system of torsion free modules and $\prod_{\gamma \in \Gamma} B(\gamma)$ the product module. If $s \in \prod_{\gamma \in \Gamma} B(\gamma)$, let

$$
\text { support } s=\{\gamma ; s(\gamma) \neq 0\}
$$

The direct sum $\bigoplus_{\gamma \in \Gamma} B(\gamma)$ is the submodule of all elements with finite support. We define

$$
v_{\min }: \bigoplus_{\gamma \in \Gamma} B(\gamma) \longrightarrow \Gamma
$$

by $v_{\min }(s)=\min (\operatorname{support} s)$ (by convention, $\left.\min \emptyset=\infty\right)$. This is a valuation, and the valued module thus obtained is the Hahn sum, denoted by $\coprod_{\gamma \in \Gamma} B(\gamma)$. The Hahn product, denoted by $\mathbf{H}_{\gamma \in \Gamma} B(\gamma)$, is the submodule of $\prod_{\gamma \in \Gamma} B(\gamma)$ consisting of all elements with well ordered support, equipped with the valuation $v_{\text {min }}$. We have

$$
S\left(\coprod_{\gamma \in \Gamma} B(\gamma)\right) \simeq[\Gamma,\{B(\gamma) ; \gamma \in \Gamma\}] \simeq S(\underset{\gamma \in \Gamma}{\mathbf{H}} B(\gamma)) .
$$

Suppose that $M_{1} \subset M_{2}$ and $\Gamma_{1} \subset \Gamma_{2}$. We will say that $\left(M_{2}, v_{2}\right)$ is an extension of $\left(M_{1}, v_{1}\right)$ and write $\left(M_{1}, v_{1}\right) \subset\left(M_{2}, v_{2}\right)$ if $v_{2}(x)=v_{1}(x)$ for all $x \in M_{1}$. In this case, for every $\gamma \in \Gamma_{1}$ there exists a natural identification of $B_{1}(\gamma)$ with a subspace of $B_{2}(\gamma)$. In this context, if $\gamma \in \Gamma_{2} \backslash \Gamma_{1}$, we set by convention: $\left(M_{1}\right)^{\gamma}=\left(M_{2}\right)^{\gamma} \cap M_{1}$, $\left(M_{1}\right)_{\gamma}=\left(M_{2}\right)_{\gamma} \cap M_{1}$ and $B_{1}(\gamma)=0$. If $\Gamma_{1}=\Gamma_{2}$ and for all $\gamma \in \Gamma_{1}, B_{1}(\gamma)=B_{2}(\gamma)$, we will say that the extension is immediate.

Remark 2.3 The extension $\left(M_{1}, v_{1}\right) \subset\left(M_{2}, v_{2}\right)$ is immediate if and only if for all $x \in M_{2}$ there exists $y \in M_{1}$ such that $v_{2}(x-y)>v_{2}(x)$. For example,

$$
\coprod_{\gamma \in \Gamma} B(\gamma) \subset \underset{\gamma \in \Gamma}{\mathbf{H}} B(\gamma)
$$

is immediate.

We will say that $M$ is maximally valued if it does not admit any proper immediate extension.

Let $\left\{x_{i} ; i \in I\right\} \subset M$ and $M_{0} \subset M$ a submodule. We will say that $\left\{x_{i} ; i \in I\right\}$ is (linearly) valuation independent over $M_{0}$ if for all $z_{0} \in M_{0}$ and $r_{i} \in R$ such that $r_{i}=0$ for almost all $i \in I$,

$$
v\left(\sum_{i \in I} r_{i} x_{i}+z_{0}\right)=\min _{\left\{i \in I ; r_{i} \neq 0\right\}}\left\{v\left(x_{i}\right), v\left(z_{0}\right)\right\} .
$$

If this is the case, then in particular, $\left\{x_{i} ; i \in I\right\}$ is linearly independent over $M_{0}$. By convention, $\emptyset$ is valuation independent over $M_{0}$. We will say that $\left\{x_{i} ; i \in I\right\}$ is (linearly) valuation independent if it is valuation independent over $M_{0}=\{0\}$. Note that this definition is given for the case of valued modules with value preserving scalar multiplication. For the general case, it is too strong and should be suitably adapted. In the theory of valued fields, there is also the notion of "algebraically valuation independent", but here we will only deal with the above defined notion, so we will omit the specification "linearly". 
Using Zorn's Lemma, it may be shown that in every valued module, there exist maximal valuation independent subsets.

$$
\text { If }\left\{x_{i} ; i \in I\right\} \subset M \text {, then } \quad{ }_{R}^{M}\left\langle\left\{x_{i} ; i \in I\right\}\right\rangle
$$

will denote the $R$-submodule of $M$ generated by the elements $x_{i}$ (by convention, ${ }_{R}^{M}\langle\emptyset\rangle=0$ ). If the context is clear, we will omit " $M$ " or " $R$ ".

In the sequel, we will assume that $R=K$ is a field, so that $M=V$ is a valued vector space with $S(V)=[\Gamma,\{B(\gamma) ; \gamma \in \Gamma\}]$. For the proofs of the facts that we will state now (Lemma 2.4 till Corollary 2.8), we refer the reader to the article [GRA1] of Gravett.

Lemma 2.4 If $\left\{x_{i} ; i \in I\right\} \subset V$ is maximal valuation independent, then

$$
{ }_{K}\left\langle\left\{x_{i} ; i \in I\right\}\right\rangle \subset V
$$

is an immediate extension, and

$$
{ }_{K}\left\langle\left\{x_{i} ; i \in I\right\}\right\rangle \simeq \coprod_{\gamma \in \Gamma} B(\gamma)
$$

as valued vector spaces.

A basis $\mathcal{B}$ of $V$ is called a valuation basis if it is a valuation independent set. In this case, $\mathcal{B}$ is maximal valuation independent (cf. Corollary 2.11 below), and since $\coprod_{\gamma \in \Gamma} B(\gamma)$ admits a valuation basis, Lemma 2.4 gives us a characterization of those vector spaces which admit a valuation basis:

Corollary 2.5 A valued vector space admits a valuation basis if and only if it is isomorphic to the Hahn sum taken over its skeleton.

The notion of valuation independence is treated in more detail in the following section.

The next theorem is central in the theory of valued vector spaces:

Theorem 2.6 Suppose that

(i) $V_{i}$ and $V_{i}^{\prime}$ are valued vector spaces and $V_{i}^{\prime}$ is an immediate extension of $V_{i}$, for $i=1,2$,

(ii) $h$ is an isomorphism of valued vector spaces of $V_{1}$ onto $V_{2}$,

(iii) $V_{2}^{\prime}$ is maximally valued.

Then there exists an embedding $h^{\prime}$ of valued vector spaces of $V_{1}^{\prime}$ into $V_{2}^{\prime}$ such that $h^{\prime}$ prolongates $h$. Moreover, $h^{\prime}$ is an isomorphism of valued vector spaces of $V_{1}^{\prime}$ onto $V_{2}^{\prime}$ if and only if $V_{1}^{\prime}$ is maximally valued.

One consequence is the following theorem which illuminates the role played by the Hahn sums and products:

Theorem 2.7 Let $\left\{x_{i} ; i \in I\right\} \subset V$ be maximal valuation independent and $h$ the isomorphism of ${ }_{K}\left\langle\left\{x_{i} ; i \in I\right\}\right\rangle$ onto $\coprod_{\gamma \in \Gamma} B(\gamma)$. Then there exists an embedding $h^{\prime}$ of $V$ into $\mathbf{H}_{\gamma \in \Gamma} B(\gamma)$ prolongating $h$. 
As a corollary, we obtain a characterization of maximally valued vector spaces. Since $\mathbf{H}_{\gamma \in \Gamma} B(\gamma)$ is maximally valued (cf. [GRA1]), the above theorem yields

Corollary 2.8 A valued vector space is maximally valued if and only if it is isomorphic to the Hahn product over its skeleton.

Also the next lemma is a consequence of the foregoing theorems:

Lemma 2.9 The following assertions are equivalent:

1) $V$ is maximally valued and admits a valuation basis

2) $\coprod_{\gamma \in \Gamma} B(\gamma) \simeq \mathbf{H}_{\gamma \in \Gamma} B(\gamma)$

3) $\coprod_{\gamma \in \Gamma} B(\gamma)=\mathbf{H}_{\gamma \in \Gamma} B(\gamma)$

4) every well ordered subset of $\Gamma$ is finite

5) $\Gamma$ is the inverse of an ordinal.

\subsection{Valuation independence}

Proposition 2.10 Let $\mathcal{B} \subset V$. Then $\mathcal{B}$ is valuation independent over $V_{0}$ if and only if the following holds: for all $n \in \mathbb{N}$ and different $b_{1}, \ldots, b_{n} \in \mathcal{B}$ with $v\left(b_{1}\right)=$ $\ldots=v\left(b_{n}\right)=\gamma$, the coefficients $\pi^{V}\left(\gamma, b_{1}\right), \ldots, \pi^{V}\left(\gamma, b_{n}\right)$ in $B(V, \gamma)$ are linearly independent over $B\left(V_{0}, \gamma\right)$.

Proof: $\quad \Rightarrow$ : Suppose there are $b_{1}, \ldots, b_{n} \in \mathcal{B}$ with $v\left(b_{1}\right)=\ldots=v\left(b_{n}\right)=\gamma$ such that $\pi^{V}\left(\gamma, b_{1}\right), \ldots, \pi^{V}\left(\gamma, b_{n}\right)$ in $B(V, \gamma)$ are not linearly independent over $B\left(V_{0}, \gamma\right)$. Then there exist nonzero $k_{1}, \ldots, k_{n} \in K$ such that $\pi^{V}\left(\gamma, \sum k_{i} b_{i}\right) \in B\left(V_{0}, \gamma\right)$. If we choose $z_{0} \in V_{0}$ satisfying $\pi^{V}\left(\gamma, z_{0}\right)=\pi^{V}\left(\gamma,-\sum k_{i} b_{i}\right)$ then we obtain

$$
v\left(k_{1} b_{1}+\ldots+k_{n} b_{n}+z_{0}\right)>\gamma=\min _{\left\{i \in I ; k_{i} \neq 0\right\}}\left\{v\left(b_{i}\right), v\left(z_{0}\right)\right\}
$$

which shows that $\mathcal{B}$ is not valuation independent over $V_{0}$.

$\Leftarrow$ : Let $\sum_{i \in I} k_{i} b_{i}$ be a finite sum of elements $b_{i} \in \mathcal{B}$ with $k_{i} \in K$ and let $z_{0} \in V_{0}$. Let $\gamma=\min _{\left\{i \in I ; k_{i} \neq 0\right\}}\left\{v\left(b_{i}\right)\right\}$. If $v\left(z_{0}\right)<\gamma$, then

$$
v\left(\sum_{i} k_{i} b_{i}+z_{0}\right)=v\left(z_{0}\right)=\min _{\left\{i \in I ; k_{i} \neq 0\right\}}\left\{v\left(b_{i}\right), v\left(z_{0}\right)\right\} .
$$

Assume now that $v\left(z_{0}\right) \geq \gamma$. Without loss of generality, let $1, \ldots, n$ be precisely the indices for which $k_{i} \neq 0$ and $v\left(b_{i}\right)=\gamma$. If $\pi^{V}\left(\gamma, b_{1}\right), \ldots, \pi^{V}\left(\gamma, b_{n}\right)$ are linearly independent over $B\left(V_{0}, \gamma\right)$, that is,

$$
\pi^{V}\left(\gamma, \sum_{i \in I} k_{i} b_{i}+z_{0}\right)=\sum_{i=1}^{n} k_{i} \pi^{V}\left(\gamma, b_{i}\right)+\pi^{V}\left(\gamma, z_{0}\right) \neq 0
$$

then

$$
v\left(\sum_{i \in I} k_{i} b_{i}+z_{0}\right)=\gamma=\min _{\left\{i \in I ; k_{i} \neq 0\right\}}\left\{v\left(b_{i}\right)\right\}=\min _{\left\{i \in I ; k_{i} \neq 0\right\}}\left\{v\left(b_{i}\right), v\left(z_{0}\right)\right\} .
$$

This proposition shows: 
Corollary 2.11 Let $\mathcal{B} \subset V$. Then $\mathcal{B}$ is maximal valuation independent over $V_{0}$ if and only if for every $\gamma \in v(V)$,

$$
\mathcal{B}_{\gamma}=\left\{\pi^{V}(\gamma, b) ; b \in \mathcal{B} \text { and } v(b)=\gamma\right\}
$$

forms a basis of $B(V, \gamma)$ over $B\left(V_{0}, \gamma\right)$.

A useful consequence of this corollary is the following well known fact. It may be used to prove by induction that every valued vector space of countable dimension admits a valuation basis (cf. $[\mathrm{BR}]$ ).

Lemma 2.12 Let $(V, v)$ be a valued $K$-vector space. If $W$ is a finite dimensional subvector space of $V$ having valuation basis $\mathcal{B}$, and if $a \in V$, then $\mathcal{B}$ can be extended to a valuation basis of $W+K a$.

Proof: The value set of $W$ is just $\{v(b) \mid b \in \mathcal{B}\}$ and thus finite. Hence, there exists some $a_{0} \in W$ such that $v\left(a-a_{0}\right) \notin v(W)$ or, if this is not possible, such that $v\left(a-a_{0}\right) \in v(W)$ is maximal. Since the case $a \in W$ is trivial, we may assume $a \notin W$ which yields that $v\left(a-a_{0}\right) \neq \infty$. If $v\left(a-a_{0}\right) \notin v(W)$, then $\mathcal{B} \cup\left\{a-a_{0}\right\}$ is the required valuation basis of $W+K a$. Now assume that $\gamma:=v\left(a-a_{0}\right) \in v(W)$. By the preceding corollary, $\mathcal{B}_{\gamma}$ forms a basis of $B(W, \gamma)$. If $\pi^{W}\left(\gamma, a-a_{0}\right)$ would lie in $B(W, \gamma)$ then there would be a linear combination $a_{1}$ of the elements in $\mathcal{B}$ with value $\gamma$ such that $\pi^{W}\left(\gamma, a-a_{0}-a_{1}\right)=0$. But this would mean that $v\left(a-a_{0}-a_{1}\right)>\gamma$, a contradiction to the maximality of $\gamma$. This shows that $\pi^{W}\left(\gamma, a-a_{0}\right)$ cannot be an element of $B(W, \gamma)$. In view of the above proposition, we find that $a-a_{0}$ is valuation independent over $W$ and again, it follows that $\mathcal{B} \cup\left\{a-a_{0}\right\}$ is the required valuation basis of $W+K a$.

This proof shows that the lemma works as well if the condition " $W$ is finite dimensional" is replaced by "every subset of $v(W)$ admits a maximal element" or equivalently, " $v(W)$ is the inverse of an ordinal" (cf. [K1]).

Valuation independent sets may serve to obtain isomorphisms between valued vector spaces, on the basis of the following lemma.

Lemma 2.13 Let $(V, v)$ and $\left(V^{\prime}, v^{\prime}\right)$ be valued $K$-vector spaces containing $\left(V_{0}, v\right)$ as a common valued subspace and such that $v(V)=v^{\prime}\left(V^{\prime}\right)$. Let $\mathcal{B} \subset V$ and $\mathcal{B}^{\prime} \subset V^{\prime}$ be valuation independent over $V_{0}$. Suppose that there exists a bijection

$$
h_{\mathcal{B}}: \mathcal{B} \longrightarrow \mathcal{B}^{\prime}
$$

such that

$$
\forall b \in \mathcal{B}: v^{\prime}\left(h_{\mathcal{B}}(b)\right)=v(b) .
$$

Then $h_{\mathcal{B}}$ extends linearly to an isomorphism over $V_{0}$

$$
h:{ }^{V}\left\langle\mathcal{B} \cup V_{0}\right\rangle \longrightarrow{ }^{V^{\prime}}\left\langle\mathcal{B}^{\prime} \cup V_{0}\right\rangle
$$

of valued vector spaces which also preserves the valuation (more precisely, which satisfies $h_{\mathrm{rk}}=\mathrm{id}$ ). 
Proof: $\quad$ We put $W={ }^{V}\left\langle\mathcal{B} \cup V_{0}\right\rangle$ and $W^{\prime}={ }^{V^{\prime}}\left\langle\mathcal{B}^{\prime} \cup V_{0}\right\rangle$. Then $\mathcal{B}$ and $\mathcal{B}^{\prime}$ are valuation bases of $W$ resp. $W^{\prime}$ over $V_{0}$, and $h: W \rightarrow W^{\prime}$ is given as follows: for every $z \in V_{0}$ and $\sum_{i \in I} k_{i} b_{i}$ a finite linear combination of elements $b_{i}$ in $\mathcal{B}$,

$$
h\left(\sum_{i \in I} k_{i} b_{i}+z\right)=\sum_{i \in I} k_{i} h_{\mathcal{B}}\left(b_{i}\right)+z .
$$

Since $\mathcal{B}$ and $\mathcal{B}^{\prime}$ are valuation bases of $W$ resp. $W^{\prime}$ over $V_{0}$, and since $v^{\prime}\left(h_{\mathcal{B}}(b)\right)=v(b)$ for every $b \in \mathcal{B}$ by hypothesis, we have

$$
\begin{aligned}
v^{\prime}\left(\sum_{i \in I} k_{i} h_{\mathcal{B}}\left(b_{i}\right)+z\right) & =\min _{\left\{i \in I ; k_{i} \neq 0\right\}}\left\{v^{\prime}\left(h_{\mathcal{B}}\left(b_{i}\right)\right), v(z)\right\} \\
& =\min _{\left\{i \in I ; k_{i} \neq 0\right\}}\left\{v\left(b_{i}\right), v(z)\right\} \\
& =v\left(\sum_{i \in I} k_{i} b_{i}+z\right),
\end{aligned}
$$

This shows that $h$ preserves the valuation, which in turn yields that $h$ is welldefined and bijective.

\subsection{Generalities about ordered modules}

In this section, let $\left(R,<_{R}\right)$ be a fixed commutative ordered ring with 1 , satisfying

$$
\forall r \in R \backslash\{0\} \exists s \in R: r s \geq 1 .
$$

Note that this condition is satisfied by every ordered field and by every archimedean ordered ring. Let $M$ be an $R$-module and $<$ a total order defined on the set $M$. We will say that $(M,<)$ is an ordered $\left(R,<_{R}\right)$-module if its underlying additive group is an ordered Abelian group, that is, if the order $<$ satisfies

1) $\forall x, y \in M: 0<x$ and $0<y \Longrightarrow 0<x+y$,

2) $x<y$ if and only if $0<y-x$,

and if the scalar multiplication satisfies

3) $\forall r \in R: 0<x$ and $0<_{R} r \Longrightarrow 0<r x$.

Simple consequences are the following rules:

if $x<y$, then $x+z<y+z$ for all $z \in M$,

if $x<y$, then $r x<r y$ for all $r \in R$ with $0<_{R} r$,

$0<x$ if and only if $-x<0$.

Every ordered $\left(R,<_{R}\right)$-module is torsion free. Every ordered Abelian group is an ordered $\mathbb{Z}$-module.

Let $\left(M_{1},<\right),\left(M_{2},<\right)$ be ordered $\left(R,<_{R}\right)$-modules and

$$
h: M_{1} \longrightarrow M_{2}
$$

a homomorphism of modules. We will say that $h$ preserves the order (or that $h$ is a homomorphism of ordered modules) if for all $x, y \in M_{1}, x \leq y$ implies $h(x) \leq h(y)$. 
A submodule $N \subset M$ is said to be convex (in $M$ ) if it satisfies: if $x_{1}, x_{2} \in N$ and $x \in M$ such that $x_{1}<x<x_{2}$, then $x \in N$. The set of all convex submodules of $M$, ordered by inclusion, is a chain containing 0 and $M$ and is closed under unions and intersections. If $N$ is convex in $M$, the quotient module $M / N$, equipped with the order induced by

$$
x \leq y \Longrightarrow x+N \leq y+N
$$

is an ordered $\left(R,<_{R}\right)$-module.

Note that if $h: M_{1} \rightarrow M_{2}$ is a surjective homomorphism of ordered modules with kernel $N$, then $N$ is convex in $M_{1}$ and $h$ induces an isomorphism of ordered modules from $M_{1} / N$ onto $M_{2}$.

For $x \in M$ we define:

$$
\begin{aligned}
& C_{x}(M)=\bigcap\{C ; C \text { is convex and } x \in C\} \\
& D_{x}(M)=\bigcup\{D ; D \text { is convex and } x \notin D\}
\end{aligned}
$$

and by convention,

$$
D_{0}(M)=\{0\}
$$

A convex submodule of $M$ is called principal if it is of the form $C_{x}(M)$ for some $x \in M$. Note that if $N$ is convex in $M$ and $x \in N$, then

$$
C_{x}(N)=C_{x}(M) \text { and } D_{x}(N)=D_{x}(M) .
$$

The ordered module

$$
B_{x}(M)=C_{x}(M) / D_{x}(M)
$$

will be called the component of $x$ in $M$. We will write $B_{x}, C_{x}$ and $D_{x}$ if the context is clear. Note that $B_{0}(M)=\{0\}$.

We put $|x|=\max \{x,-x\}$. Let $y \in M$; we will say that $x$ is $R$-equivalent to $y$ and write $x \stackrel{R}{\sim} y$ if there exists $r \in R$ such that

$$
r|x| \geq|y| \text { and } r|y| \geq|x|
$$

We will say that $x$ is $R$-infinitely smaller than $y$ and write $x \stackrel{R}{\ll} y$ if $r|x|<|y|$ for all $r \in R$. We remark the following properties: $\stackrel{R}{\sim}$ is an equivalence relation, and $\stackrel{R}{\ll}$ is compatible with this equivalence relation:

$$
\begin{aligned}
& x \stackrel{R}{\ll} y \text { and } x \stackrel{R}{\sim} z \Longrightarrow z \stackrel{R}{\ll} y \\
& x \stackrel{R}{\ll} y \text { and } y \stackrel{R}{\sim} z \Longrightarrow x \stackrel{R}{\ll} z .
\end{aligned}
$$

The equivalence class of $x$ will be denoted by $[x]_{R}$, and the set of equivalence classes by $\Gamma$. We define on $\Gamma$ an order in the following way:

$$
[y]_{R}<[x]_{R} \text { if and only if } x \stackrel{R}{\ll} y .
$$

By the above mentioned properties, $\Gamma$ is a chain with last element $[0]_{R}$ which we will denote by $\infty$. From condition (2) on the ring $R$ it follows that for every $r \in R$ we have $r x \stackrel{R}{\sim} x$. Now the proof of the following proposition is straightforward: 
Proposition 2.14 The map

$$
\begin{aligned}
v^{R}: M & \longrightarrow \Gamma \\
x & \mapsto[x]_{R}
\end{aligned}
$$

is a valuation on $M$.

We will call $v^{R}$ the $R$-natural valuation on $M$. Whenever we consider an ordered module as a valued module it will be understood that the valuation is the $R$-natural valuation, unless otherwise stated. Note that a given map $v$ from $M$ onto some chain is a valuation and equivalent to the $R$-natural valuation of $M$ if and only if

$$
v(x)>v(y) \Longleftrightarrow x \stackrel{R}{\ll} y \text { for all } x, y \in M .
$$

Let $v$ be any valuation on an ordered module $M$; $v$ is called compatible with the order on $M$ if for all $x, y \in M$ with $x>0$ and $y>0, v(x)<v(y)$ implies $y<x$. In particular, $v^{R}$ is compatible with the order on $M$.

We will say that $M$ is $R$-archimedean if $\left(M, v^{R}\right)$ is homogeneous, that is, $v^{R}(M)$ contains only one element, apart from $\infty$. If $x \in M$ with $v^{R}(x)=\gamma$, then $C_{x}=M^{\gamma}$ and $D_{x}=M_{\gamma}$; in particular, $M$ is $R$-archimedean if and only if $M$ does not contain any nontrivial convex submodule. Hence, $B_{x}=B(\gamma)=C_{x} / D_{x}$ is an $R-$ archimedean ordered $\left(R,<_{R}\right)$-module, and the homomorphism $\pi(\gamma,-)$ preserves the order. To indicate the existence of the induced order on the components, we will speak of the ordered skeleton of $M$ and write $S(M,<)$, and we will call $B(\gamma)$ the $R$-archimedean component corresponding to $\gamma$. Similarly, we will say that $S\left(M_{1},<\right)$ and $S\left(M_{2},<\right)$ are isomorphic, or that $S\left(M_{1}\right)$ and $S\left(M_{2}\right)$ are isomorphic as ordered skeletons if there exists an isomorphism

$$
\varphi: \Gamma_{1} \longrightarrow \Gamma_{2}
$$

of chains, and for every $\gamma \in \Gamma_{1}$ an isomorphism

$$
\varphi_{\gamma}: B\left(M_{1}, \gamma\right) \longrightarrow B\left(M_{2}, \varphi(\gamma)\right)
$$

of ordered modules. And so on for all other notions defined in section 2.1.

If $M$ and $N$ are ordered modules, then $M \amalg N$ denotes the sum $M \oplus N$ equipped with the lexicographic order. We will not distinguish between external and internal sums, but we will occasionally indicate internal sums by writing " $M$ ' $=M \amalg N$ " instead of " $M$ ' $\simeq M \amalg N$ ".

Lemma 2.15 a) Let $M$ be an ordered module, $C$ a convex submodule of $M$ and $C^{\prime}$ a complement to $C$ in $M$, i.e. $C^{\prime}$ is a submodule of $M$ such that $M=C^{\prime} \oplus C$. Then $M \simeq C^{\prime} \amalg C$ as ordered modules.

b) Let $\eta: M \rightarrow N$ a surjective homomorphism of ordered modules and assume that ker $\eta$ has a complement in $M$. Then

$$
M \simeq N \amalg \operatorname{ker} \eta \text {. }
$$


Proof: a) It is easy to verify that

$$
\begin{aligned}
\psi: M=C^{\prime} \oplus C & \longrightarrow C^{\prime} \amalg C \\
b+c & \mapsto \quad(b, c)
\end{aligned}
$$

is an isomorphism of ordered modules.

b) Let $C^{\prime}$ be a submodule of $M$ such that

$$
M=C^{\prime} \oplus \operatorname{ker} \eta
$$

( $C$ ' exists by hypothesis), hence by a),

$$
M=C^{\prime} \amalg \operatorname{ker} \eta
$$

(ker $\eta$ being convex), and

$$
\eta \uparrow^{\prime} C^{\prime}: C^{\prime} \longrightarrow N
$$

is an isomorphism of ordered modules.

For the computation of skeletons, there is an analogue to part b) of the preceding lemma which has the advantage that it will not require the existence of a complement to ker $\eta$ in $M$. We first need some notations. If $\Delta_{1}, \Delta_{2}$ are two chains, then $\Delta_{1}+\Delta_{2}$ will denote their sum, that is, the set $\left(\{1\} \times \Delta_{1}\right) \cup\left(\{2\} \times \Delta_{2}\right)$ ordered lexicographically. Now let $S_{i}=\left[\Delta_{i},\left\{B_{i}(\delta) ; \delta \in \Delta_{i}\right\}\right]$ be ordered systems of modules, for $i=1,2$. Then $S_{1} \amalg S_{2}$, called the sum of $S_{1}$ and $S_{2}$, will denote the ordered system

$$
\left[\Delta_{1}^{-}+\Delta_{2},\left\{A(\gamma) ; \gamma \in \Delta_{1}^{-}+\Delta_{2}\right\}\right]
$$

where

$$
A(\gamma)= \begin{cases}B_{1}(\delta) & \text { if } \gamma=(1, \delta) \in\{1\} \times \Delta_{1}^{-} \\ B_{2}(\delta) & \text { if } \gamma=(2, \delta) \in\{2\} \times \Delta_{2}\end{cases}
$$

Lemma 2.16 Let $\eta: M \rightarrow N$ be a surjective homomorphism of ordered modules. Then

$$
S(M) \simeq S(N) \amalg S(\operatorname{ker} \eta)
$$

More precisely, if $v$ and $v^{\prime}$ are the natural valuations of $M$ and $N$ respectively and the restriction of $v$ to $\operatorname{ker} \eta$ is again denoted by $v$, then the map

$$
w: M \rightarrow v^{\prime}(N)^{-}+v(\operatorname{ker} \eta)
$$

given by

$$
w(a)= \begin{cases}v^{\prime}(\eta(a)) & \text { if } a \notin \operatorname{ker} \eta \\ v(a) & \text { if } a \in \operatorname{ker} \eta\end{cases}
$$

is welldefined, surjective and equivalent to the natural valuation $v$ on $M$. Further,

$$
\begin{aligned}
& B_{a}(M) \simeq B_{\eta(a)}(N) \quad \text { if } a \notin \operatorname{ker} \eta \\
& B_{a}(M)=B_{a}(\operatorname{ker} \eta) \text { if } a \in \operatorname{ker} \eta
\end{aligned}
$$


Proof: $\quad$ Since the epimorphism $\eta$ preserves $\leq$ (that is, $a \leq b \Rightarrow \eta(a) \leq \eta(b)$ ), its kernel is a convex submodule of $M$. It also yields that $\eta$ sends convex submodules of $M$ to convex submodules of $N$, and that the preimage of a convex submodule of $N$ is a convex submodule of $M$. If $C_{1} \supset C_{2} \supset$ ker $\eta$ are convex submodules of $M$ such that $\eta\left(C_{1}\right)=\eta\left(C_{2}\right)$, then $C_{1}=C_{2}+$ ker $\eta=C_{2}$. We have thus proved that $C \mapsto \eta(C)$ is a bijective correspondence of the convex submodules of $M$ containing ker $\eta$ to the convex submodules of $N$. The set of convex submodules of $M$ is linearly ordered by inclusion, so the convex submodules which do not contain ker $\eta$ are themselves contained in ker $\eta$. Consequently, the convex submodules of $M$ not containing ker $\eta$ are precisely the convex submodules of ker $\eta$.

Everything that we have said holds as well for the principal convex submodules. Since the correspondence $C_{x} \mapsto v(x)$ is an order reversing bijection from the set of principal convex submodules of an ordered module onto its value set, our above considerations prove that the valuation $w$ defined by (3) is equivalent to the natural valuation $v$ on $M$.

By what we have said about the convex submodules of ker $\eta$, it is clear that $B(M, \gamma)=B(\operatorname{ker} \eta, \gamma)$ whenever $\gamma \in v(\operatorname{ker} \eta)$. If $a \notin \operatorname{ker} \eta$, then the minimal convex submodule $C_{a}$ of $M$ containing $a$, properly contains ker $\eta$. The maximal convex submodule $D_{a}$ of $M$ not containing $a$, will then also contain ker $\eta$, and thus we may compute

$$
B(M, v(a))=C_{a} / D_{a} \simeq\left(C_{a} / \operatorname{ker} \eta\right) /\left(D_{a} / \operatorname{ker} \eta\right) \simeq \eta\left(C_{a}\right) / \eta\left(D_{a}\right) .
$$

By the correspondence that we have described at the beginning of this proof, it follows that $\eta\left(C_{a}\right)$ is the least convex submodule of $N$ containing $\eta(a)$ and that $\eta\left(D_{a}\right)$ is the largest convex submodule of $N$ not containing $\eta(a)$. This shows that $\eta\left(C_{a}\right) / \eta\left(D_{a}\right)=B(N, v(\eta(a)))$ which completes our proof.

Since every convex submodule $C$ of $M$ appears as a kernel of some homomorphism (namely, the canonical epimorphism $M \rightarrow M / C$ ), the above lemma may also be read as a lemma about convex submodules. In the lexicographic sum $A \amalg B$ of ordered modules, the submodule $B$ is convex; hence, the lemma also shows that the skeleton of a lexicographic sum $A \amalg B$ is (isomorphic to) the lexicographic sum $S(A) \amalg S(B)$ of their skeletons. This can even be shown for infinite lexicographic sums.

The following two lemmas will be used in the present and the subsequent paper [K-K1]. Their proofs rely significantly on the foregoing lemma.

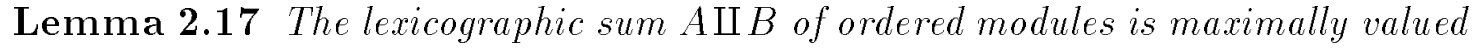
if and only if both $A$ and $B$ are.

Proof: $\Rightarrow$ : If $B$ is not maximally valued, then there is an immediate extension $B^{\prime}$ of $B$, and by virtue of the foregoing lemma, also $A \amalg B^{\prime}$ will be an immediate extension of $A \amalg B$. A similar argument works if $A$ is not maximally valued.

$\Leftarrow$ : Let $M$ be a nontrivial extension of $A \amalg B$. Then the convex hull $B^{\prime}$ of $B$ in $M$ is an extension of $B$ and the canonical epimorphism $M \rightarrow M / B^{\prime}$ induces an embedding of $A$ into $M / B^{\prime}$. If both $A$ and $B$ are maximally valued, then at least one of the extensions $B \subset B^{\prime}$ and $A \subset M / B^{\prime}$ is not immediate, and it follows from the foregoing lemma that $A \amalg B \subset M$ cannot be immediate. 
Lemma 2.18 The lexicographic sum A $\amalg B$ of ordered modules admits a valuation basis if and only if both $A$ and $B$ do.

Proof: $\quad$ Set $M=A \amalg B$ and denote by $\eta$ the canonical epimorphism onto $A$ with kernel $B$ :

$$
\begin{aligned}
\eta: M & \longrightarrow A \\
a+b & \mapsto a \quad \text { for all } a \in A, b \in B .
\end{aligned}
$$

We shall use the notation of Lemma 2.16, with $A$ in the place of $N$. The following fact will be used in the proof: since $B$ is convex in $M$, we have for every $x \in M$ :

$$
x \in B \text { if and only if } v(x) \geq v(b) \text { for some } b \in B \text {. }
$$

$\Rightarrow$ : Let $\left\{x_{i} ; i \in I\right\}$ be a valuation basis for $M$ and set

$$
\mathcal{B}=\left\{x_{i} ; i \in I\right\} \cap B .
$$

If $b \in B$ and $b=\sum_{i \in I} r_{i} x_{i}$ (where all but a finite number of the $r_{i}$ 's is zero), then

$$
v(b)=\min _{\left\{i \in I ; r_{i} \neq 0\right\}} v\left(x_{i}\right) .
$$

Consequently, for all $i \in I$ with $r_{i} \neq 0, v\left(x_{i}\right) \geq v(b)$, so by (4), $x_{i} \in B$. This shows that $\mathcal{B}$ is a valuation basis of $B$.

Now let $\mathcal{A}=\eta\left(\left\{x_{i} ; i \in I\right\}\right) \backslash\{0\}$. Clearly, $\mathcal{A}$ is a generating set for $A$. Moreover, it is valuation independent. Indeed, if $\sum r_{i} \eta\left(x_{i}\right)$ is a finite linear combination of elements of $\mathcal{A}$, then

$$
v^{\prime}\left(\sum r_{i} \eta\left(x_{i}\right)\right)=v^{\prime}\left(\eta\left(\sum r_{i} x_{i}\right)\right)
$$

since $\eta$ is a homomorphism. On the other hand, in this last sum, $\eta\left(x_{i}\right) \neq 0$ (by definition of $\mathcal{A})$, so $x_{i} \notin B$. Consequently, $v\left(\sum r_{i} x_{i}\right)=\min _{i} v\left(x_{i}\right)<v(b)$ for all $b \in B$ (by (4)). It follows (again by (4)) that $\sum r_{i} x_{i} \notin B$. Now we can apply Lemma 2.16 to obtain that

$$
v^{\prime}\left(\eta\left(\sum r_{i} x_{i}\right)\right)=w\left(\sum r_{i} x_{i}\right)=\min _{i} w\left(x_{i}\right)=\min _{i} v^{\prime}\left(\eta\left(x_{i}\right)\right),
$$

which proves the assertion.

$\Leftarrow$ : Let $\mathcal{A}(\operatorname{resp} . \mathcal{B})$ be a valuation basis of $A$ (resp. of $B)$. Then $\mathcal{A} \cup \mathcal{B}$ is a basis of $M$. Moreover, if $\sum_{i \in I} r_{i} a_{i}\left(\operatorname{resp} . \sum_{j \in J} s_{j} b_{j}\right)$ is a nontrivial finite linear combination of elements of $\mathcal{A}$ (resp. of $\mathcal{B}$ ), then by Lemma 2.16 ,

$$
w\left(\sum_{i \in I} r_{i} a_{i}\right)<w\left(\sum_{j \in J} s_{j} b_{j}\right),
$$

so

$$
w\left(\sum_{i \in I} r_{i} a_{i}+\sum_{j \in J} s_{j} b_{j}\right)=w\left(\sum_{i \in I} r_{i} a_{i}\right)=v^{\prime}\left(\sum_{i \in I} r_{i} a_{i}\right)=\min _{i} v^{\prime}\left(a_{i}\right)=\min _{i} w\left(a_{i}\right)
$$


where the last two equalities hold by assumption and Lemma 2.16. It follows that $\mathcal{A} \cup \mathcal{B}$ is valuation independent.

Let us now consider isomorphisms of ordered modules. If $h$ is such an isomorphism, then it preserves the valuation and induces an isomorphism of the ordered skeletons. But it is the converse to this statement which is important for us:

Proposition 2.19 Suppose that $M_{1}$ and $M_{2}$ are ordered modules and that

$$
h: M_{1} \longrightarrow M_{2}
$$

is an isomorphism of valued modules. If

$$
\left[h_{\mathrm{rk}},\left\{h_{\gamma} ; \gamma \in \Gamma_{1}\right\}\right]: S\left(M_{1}\right) \simeq S\left(M_{2}\right)
$$

is an isomorphism of ordered skeletons, then $h$ preserves the order.

Proof: $\quad$ Let $x>0$ and put $\gamma=v^{R}(x)$. Then $\pi^{M_{1}}(\gamma, x)>0\left(\right.$ in $B\left(M_{1}, \gamma\right)$ ), hence $h_{\gamma}\left(\pi^{M_{1}}(\gamma, x)\right)>0$. That is, $\pi^{M_{2}}\left(h_{\mathrm{rk}}(\gamma), h(x)\right)>0\left(\right.$ in $\left.B\left(M_{2}, h_{\mathrm{rk}}(\gamma)\right)\right)$, whence $h(x)>0$.

As a corollary, we obtain the following lemma due to R. A. H. Gravett ([GRA2], Lemme 1). Gravett states it for the case $R=\mathbb{Q}$, but the lemma is true for arbitrary ordered $R$-modules.

Lemma 2.20 Suppose that

(i) $M_{i}$ and $M_{i}^{\prime}$ are ordered $R$-modules, for $i=1,2$,

(ii) $M_{i} \subset M_{i}^{\prime}$ are immediate extensions, for $i=1,2$,

(iii) $h^{\prime}: M_{1}^{\prime} \rightarrow M_{2}^{\prime}$ is an isomorphism of valued $R$-modules, and $h^{\prime} \uparrow M_{1}: M_{1} \rightarrow$ $M_{2}$ is an isomorphism of ordered $R$-modules.

Then $h^{\prime}$ is an isomorphism of ordered $R$-modules.

Proof: $\quad$ Since $h^{\prime} \uparrow M_{1}: M_{1} \rightarrow M_{2}$ preserves the order and $M_{i} \subset M_{i}^{\prime}$ are immediate extensions, we have that

$$
\left[h_{\mathrm{rk}}^{\prime},\left\{h_{\gamma}^{\prime} ; \gamma \in \Gamma_{1}\right\}\right]: S\left(M_{1}^{\prime}\right) \simeq S\left(M_{2}^{\prime}\right)
$$

is an isomorphism of ordered skeletons, hence $h^{\prime}: M_{1}^{\prime} \rightarrow M_{2}^{\prime}$ preserves the order by virtue of the foregoing proposition.

We want to exploit this lemma to obtain the analogues to Corollary 2.5, Theorem 2.6, Corollary 2.8 and Theorem 2.7 in the case of ordered vector spaces. To this end, we need the following definitions. Let $[\Gamma,\{B(\gamma) ; \gamma \in \Gamma\}]$ be a system of $R$-archimedean ordered $\left(R,<_{R}\right)$-modules. On $\bigoplus_{\gamma \in \Gamma} B(\gamma)$, we define the lexicographical order $<_{l}$ :

for all $s_{1}, s_{2} \in \bigoplus_{\gamma \in \Gamma} B(\gamma), s_{1}<_{l} s_{2}$ if $s_{1}(\gamma)<s_{2}(\gamma)$ for $\gamma=\min \operatorname{support}\left(s_{1}-s_{2}\right)$. The ordered $\left(R,<_{R}\right)$-module obtained in this way is called the lexicographical sum. Similarly, the lexicographical product is the submodule of $\prod_{\gamma \in \Gamma} B(\gamma)$ consisting of 
all elements with wellordered support, equipped with the order $<_{l}$. Then for all $s_{1}, s_{2}$,

$$
s_{1} \stackrel{R}{\sim} s_{2} \text { if and only if min support } s_{1}=\min \text { support } s_{2} .
$$

Hence, as valued $\left(R,<_{R}\right)$-module, the lexicographical sum (resp. the lexicographical product) is isomorphic to the Hahn sum (resp. Hahn product), and we will also denote it by $\coprod_{\gamma \in \Gamma} B(\gamma)$ (resp. $\left.\mathbf{H}_{\gamma \in \Gamma} B(\gamma)\right)$. We have

$$
S\left(\coprod_{\gamma \in \Gamma} B(\gamma)\right) \simeq[\Gamma,\{B(\gamma) ; \gamma \in \Gamma\}] \simeq S(\underset{\gamma \in \Gamma}{\mathbf{H}} B(\gamma))
$$

as ordered skeletons.

From now on, we will consider an ordered field $\left(K,<_{K}\right)$ and an ordered $K-$ vector space $(V,<)$. In this situation, we have the analogue to Corollary 2.5:

Proposition 2.21 An ordered vector space admits a valuation basis if and only if it is isomorphic (as ordered vector space) to the lexicographical sum over its ordered skeleton.

Proof: Only " $\Rightarrow$ " is nontrivial. Let $\mathcal{B}=\left\{x_{i} ; i \in I\right\}$ be a valuation basis of $(V,<)$ and let

$$
h: V \longrightarrow \coprod_{\gamma \in \Gamma} B(V, \gamma)
$$

be the map given by $x_{i} \mapsto s_{i}$, where the tuple $s_{i}$ is defined by

$$
s_{i}(\gamma)=\left\{\begin{array}{cl}
\pi^{V}\left(\gamma, x_{i}\right) & \text { if } \gamma=v^{K}\left(x_{i}\right) \\
0 & \text { otherwise, }
\end{array}\right.
$$

and

$$
h\left(\sum k_{i} x_{i}\right)=\sum k_{i} s_{i} \text { for } k_{i} \in K .
$$

(Here, $h$ is the isomorphism of valued vector spaces whose existence is stated in Corollary 2.5.) Calculating $h_{\mathrm{rk}}$ and $\left\{h_{\gamma} ; \gamma \in \Gamma\right\}$, one easily verifies that

$$
\left[h_{\mathrm{rk}},\left\{h_{\gamma} ; \gamma \in \Gamma\right\}\right]: S(V) \simeq S\left(\coprod_{\gamma \in \Gamma} B(V, \gamma)\right)
$$

is an isomorphism of ordered skeletons. Hence by virtue of Proposition 2.19, $h$ preserves the order.

As a corollary to Theorem 2.6 and Lemma 2.20, we obtain:

Theorem 2.22 Suppose that

(i) $V_{i}$ and $V_{i}^{\prime}$ are ordered vector spaces and $V_{i}^{\prime}$ is an immediate extension of $V_{i}$, for $i=1,2$,

(ii) $h: V_{1} \rightarrow V_{2}$ is an isomorphism of ordered vector spaces,

(iii) $V_{2}^{\prime}$ is maximally valued.

Then there exists an embedding $h^{\prime}: V_{1}^{\prime} \rightarrow V_{2}^{\prime}$ of ordered vector spaces such that $h^{\prime}$ extends $h$. Moreover, $h^{\prime}$ is an isomorphism of ordered vector spaces if and only if $V_{1}^{\prime}$ is maximally valued. 
As a corollary, we obtain the analogue to Theorem 2.7:

Theorem 2.23 Let $\left\{x_{i} ; i \in I\right\} \subset V$ a maximal valuation independent subset, and $h:{ }_{K}\left\langle\left\{x_{i} ; i \in I\right\}\right\rangle \rightarrow \coprod_{\gamma \in \Gamma} B(V, \gamma)$ an isomorphism of ordered vector spaces. Then there exists an embedding $h^{\prime}: V \rightarrow \mathbf{H}_{\gamma \in \Gamma} B(V, \gamma)$ of ordered vector spaces, extending $h$.

As well, we obtain the analogue to Corollary 2.8:

Corollary 2.24 An ordered vector space is maximally valued if and only if it is isomorphic (as ordered vector space) to the lexicographical product taken over its ordered skeleton.

\section{$2.4 L_{\infty \omega}-$ equivalence of ordered vector spaces}

Let $L$ be an arbitrary first order language, $\mathcal{A}, \mathcal{B} L$-structures and $\mathcal{A}_{0}$ a common substructure (cf. [C-K] for these notions). We refer the reader to [BAR] or [POI] for the definitions of the infinitary language $L_{\infty}$, the infinitary equivalence of $\mathcal{A}$ and $\mathcal{B}$ over $\mathcal{A}_{0}$ (which we will indicate by " $\mathcal{A} \equiv_{\infty}$ w $\mathcal{B}$ over $\mathcal{A}_{0}$ "), the local (or partial) isomorphism, the karpian family, as well as for the main results concerning these notions.

Following the notation in $[\mathrm{BAR}]$, we will write

$$
I: \mathcal{A} \simeq_{p} \mathcal{B}\left(\text { over } \mathcal{A}_{0}\right)
$$

if $I$ is a nonempty karpian family of isomorphisms (over $\mathcal{A}_{0}$ ) of substructures of $\mathcal{A}$ onto substructures of $\mathcal{B}$.

Let us state here two results (Theorem 2 in [BAR] and Theorem 5.02 in [POI]) of which we will make constant use in this paper. For the notion of $\kappa$-saturation appearing in the second theorem, see [POI].

Theorem 2.25 If $\mathcal{A}$ and $\mathcal{B}$ are countable or countably generated structures, then $\mathcal{A} \simeq \mathcal{B}$ if and only if $\mathcal{A} \simeq_{p} \mathcal{B}$. In fact, if $I: \mathcal{A} \simeq_{p} \mathcal{B}$ and $f_{0} \in I$ then $f_{0}$ can be extended to an isomorphism $f: \mathcal{A} \simeq \mathcal{B}$.

Theorem 2.26 If $\mathcal{A}$ and $\mathcal{B}$ are elementarily equivalent and $\aleph_{0}$-saturated, then $\mathcal{A} \equiv{ }_{\infty} \mathcal{B}$.

For the proof of the next theorem, see [K1] or [K2]. The second assertion of part a) which was proved by R. Brown (cf. $[B R]$ ), is a special case of the first assertion by virtue of the preceding theorem.

Theorem 2.27 Let $K$ be an ordered field and $V_{1}, V_{2}$ two ordered $K$-vector spaces. a)

$$
V_{1} \equiv_{\infty} V_{2} \quad \text { (as ordered } K \text {-vector spaces) }
$$

if and only if

$$
S\left(V_{1}\right) \equiv_{\infty \mathrm{w}} S\left(V_{2}\right) \text { (as ordered skeletons). }
$$

If moreover $\operatorname{dim}_{K} V_{i} \leq \aleph_{0}$ for $i=1,2$, then

$$
V_{1} \simeq V_{2} \quad \text { (as ordered } K \text {-vector spaces) }
$$


if and only if

$$
S\left(V_{1}\right) \simeq S\left(V_{2}\right) \text { (as ordered skeletons). }
$$

b) Suppose that $S\left(V_{1}\right) \equiv_{\infty} S\left(V_{2}\right)$ as ordered skeletons. Then $\Gamma_{1} \equiv_{\infty} \Gamma_{2}$ as chains, and for all $\gamma \in \Gamma_{1}$ there is some $\delta \in \Gamma_{2}$ such that $B_{1}(\gamma) \equiv_{\infty} B_{2}(\delta)$ as ordered $K$-vector spaces.

\section{Applications to exponential fields}

In this chapter, we will deal with ordered Abelian groups (which are appearing in connection with exponential fields). They may be viewed as ordered $\mathbb{Z}$-modules,

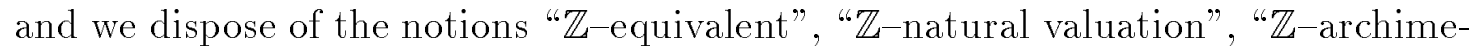
dean" and "ordered skeleton" introduced in section 2.3. Since there is no danger of confusion, we will abbreviate the terminology by omitting the " $\mathbb{Z}$ ". For example, an ordered Abelian group is "archimedean" if it does not contain proper nontrivial convex subgroups, and we have

Theorem 3.1 Every archimedean group is isomorphic (as an ordered group) to a subgroup of $(\mathbb{R},+, 0,<)$.

(Cf. Fuchs [FU] for a proof.) Hence, the skeleton of an ordered Abelian group is an ordered system of subgroups of $\mathbb{R}$.

\subsection{The natural valuation of an ordered field}

Let $K$ be a field, $G$ an ordered Abelian group and $\infty$ an element greater than every element of $G$. A surjective map

$$
v: K \longrightarrow G \cup\{\infty\}
$$

is a valuation on $K$ if and only if for all $a, b \in K$,

(i) $v(a)=\infty$ if and only if $a=0$

(ii) $v(a b)=v(a)+v(b)$

(iii) $v(a+b) \geq \min \{v(a), v(b)\}$.

As immediate consequences, we have:

$v(a)=v(-a)$

$v\left(a^{-1}\right)=-v(a)$ for $a \neq 0$

$v(a) \neq v(b) \Rightarrow v(a+b)=\min \{v(a), v(b)\}$.

We write $G=v\left(K^{\times}\right)$and call $G$ the value group of $K$. The valuation ring is the ring

$$
R_{v}=\{a ; a \in K \text { and } v(a) \geq 0\},
$$

and the valuation ideal is its maximal ideal

$$
I_{v}=\{a ; a \in K \text { and } v(a)>0\} .
$$

The field $R_{v} / I_{v}$, denoted by $\bar{K}$, is the residue field. The group of units of the valuation ring is the subgroup of the multiplicative group of $R_{v}$ defined by

$$
\mathcal{U}_{v}=\{a ; a \in K \text { and } v(a)=0\} .
$$


Let now $(K,+, \cdot, 0,1,<)$ be an ordered field. The set of positive elements of $K$ will be denoted by $K^{>0}$. Then $(K,+, 0,<)$ and $\left(K^{>0}, \cdot, 1,<\right)$ are ordered Abelian groups, and $(K,+, 0,<)$ is divisible. Consider the ordered set $G$ of equivalence classes $[a]$, for $a \in K, a \neq 0$, of the equivalence relation " $\mathbb{Z}$-equivalent" ("archimedean equivalent" in the usual terminology) defined on the divisible ordered Abelian group $(K,+, 0,<)$, the order on $G$ given by

$$
[a]<[b] \text { if and only if } b \ll a \text {. }
$$

On $G$, we define the addition: $[a]+[b]=[a b]$; equipped with this addition and order, $G$ becomes an ordered Abelian group with neutral element [1], and the natural valuation on the divisible ordered Abelian group $(K,+, 0,<)$

$$
\begin{aligned}
v: K & \longrightarrow G \cup\{\infty\} \\
a & \mapsto[a]
\end{aligned}
$$

is a valuation of the field $K$ (which we call the natural valuation on the ordered field $K$ ). It is compatible with the order, which means that

$$
\text { if } a>0 \text { and } b>0 \text {, then } v(a)<v(b) \Rightarrow b<a \text {. }
$$

In this case, $R_{v}$ and $I_{v}$ are convex in $K$; as ordered (additive) groups, $R_{v}$ and $I_{v}$ are just $C_{1}$ (the smallest convex subgroup containing 1 ) and $D_{1}$ (the largest convex subgroup not containing 1 ) respectively. Hence, $\bar{K}$ equipped with the canonical order is an archimedean ordered field, and $\bar{K}$ is just the divisible ordered Abelian group $C_{1} / D_{1}$ equipped with the multiplication

$$
\left(a+D_{1}\right) \cdot\left(b+D_{1}\right)=a b+D_{1} .
$$

The coefficient map corresponding to $v(1)$

$$
\pi(v(1),-): R_{v} \longrightarrow \bar{K}
$$

is a homomorphism of ordered rings, and it is just the residue map of the valued field $(K, v)$. To abbreviate the notation, we will denote $\pi(v(1), a)$ by $\bar{a}$ and omit "corresponding to $v(1)$ ". We have:

$$
\forall a, b \in R_{v}: \bar{a}>\bar{b} \Rightarrow a>b \text { and } a>b \Rightarrow \bar{a} \geq \bar{b}
$$

In this paper, we will always consider the natural valuation $v$ on an ordered field (and we will use (6) without explicit mentioning). Hence, $R_{v}$ and $I_{v}$ are always convex subgroups of $(K,+, 0,<)$. As a field, $K$ has multiplication, and an easy argument then shows that all archimedean components are isomorphic:

Lemma 3.2 The archimedean components $(\neq 0)$ of the divisible ordered Abelian group $(K,+, 0,<)$ are all isomorphic to the divisible ordered Abelian group $(\bar{K},+, 0,<$ ) .

Proof: $\quad$ Let $a \in K, a>0$. The map

$$
\begin{aligned}
C_{a} & \rightarrow \bar{K} \\
x & \mapsto \overline{x a^{-1}}
\end{aligned}
$$

is a surjective homomorphism of ordered groups with kernel $D_{a}$. 
Remark 3.3 An isomorphism of ordered fields preserves the natural valuation and induces isomorphisms of the corresponding residue field and value group. Hence, $\bar{K}$ and $G$ are invariants for an ordered field.

The divisibility of $(K,+, 0,<)$ enables us to present it as a lexicographic sum of three summands which will play an independent role in the course of this paper.

By the the convexity of $R_{v}$ and $I_{v}$, Lemmas 2.15, 2.16 and 3.2, we obtain:

Lemma 3.4 There exist a group complement $\mathbf{A}$ to $R_{v}$ in $(K,+, 0,<)$ and a group complement $\mathbf{A}^{\prime}$ to $I_{v}$ in $R_{v}$ such that

$$
(K,+, 0,<)=\mathbf{A} \amalg \mathbf{A}^{\prime} \amalg I_{v} .
$$

Both $\mathbf{A}$ and $\mathbf{A}^{\prime}$ are unique up to order preserving isomorphism, and $\mathbf{A}^{\prime}$ is isomorphic to the archimedean group $(\bar{K},+, 0,<)$. Furthermore, the value set of $\mathbf{A}$ is $G^{<0} \cup\{\infty\}$, the one of $I_{v}$ is $G^{>0} \cup\{\infty\}$, and the nonzero components of $\mathbf{A}$ and $I_{v}$ are all isomorphic to $(\bar{K},+, 0,<)$.

\subsection{The skeleton of $\left(K^{>0}, \cdot, 1,<\right)$}

For the multiplicative group $\left(K^{>0}, \cdot, 1,<\right)$ of positive elements, we will now derive a similar decomposition as we have done for the additive group (cf. Theorem 3.8 below). But the multiplicative group is in general not divisible. So, as a hypothesis in Theorem 3.8 we will require the divisibility which actually is equivalent to the property that $K$ is root closed for positive elements (for every $a \in K, a>0$, and for every $n \in \mathbb{N}$, there is some $b \in K$ such that $b^{n}=a$ ). Note that every real closed field has this property.

Let us consider the following subgroups of $\mathcal{U}_{v}$ : the group of positive units

$$
\mathcal{U}_{v}^{>0}=\{a ; a>0 \text { and } v(a)=0\},
$$

and the group of 1-units

$$
1+I_{v}=\{a ; v(a-1)>0\} .
$$

\section{Remark 3.5}

(i) By (5), for every $a>1$,

$$
a \in R_{v} \Longleftrightarrow a \in \mathcal{U}_{v}
$$

(ii) For all $a, b \in K, v(a-b)>v(a)$ implies $\operatorname{sign}(a)=\operatorname{sign}(b)$.

(iii) For every $a \in 1+I_{v}$ we have $v(a-1)=v\left(a^{-1}-1\right)$.

(iv) If $a \in \mathcal{U}_{v} \backslash\left(1+I_{v}\right)$, then $a^{-1} \in \mathcal{U}_{v} \backslash\left(1+I_{v}\right)$.

The following two lemmas will give information on the summands of a lexicographic decomposition of $\left(K^{>0}, \cdot, 1,<\right)$ (cf. Theorem 3.8) in the case where this group is divisible. But the lemmas are true without this hypothesis and will yield a decomposition of the skeleton of $\left(K^{>0}, \cdot, 1,<\right)$ in any case. In view of $(5)$, we have: 
Lemma 3.6 The map

$$
\begin{aligned}
\left(K^{>0}, \cdot, 1,<\right) & \longrightarrow G \\
a & \mapsto-v(a)=v\left(a^{-1}\right)
\end{aligned}
$$

is a surjective homomorphism of ordered groups with kernel $\mathcal{U}_{v}^{>0}$. It follows that $\mathcal{U}_{v}^{>0}$ is convex in $\left(K^{>0}, \cdot, 1,<\right)$ and that

$$
\left(K^{>0}, \cdot, 1,<\right) / \mathcal{U}_{v}^{>0} \simeq G
$$

as ordered groups.

Next, we consider $\mathcal{U}_{v}^{>0}$ :

Lemma 3.7 The map

$$
\begin{aligned}
\left(\mathcal{U}_{v}^{>0}, \cdot, 1,<\right) & \longrightarrow\left(\bar{K}^{>0}, \cdot, 1,<\right) \\
a & \mapsto \bar{a}
\end{aligned}
$$

is a surjective homomorphism of ordered groups with kernel $1+I_{v}$. It follows that $1+I_{v}$ is convex in $\left(\mathcal{U}_{v}^{>0}, \cdot, 1,<\right)$, and

$$
\left(\mathcal{U}_{v}^{>0}, \cdot, 1,<\right) / 1+I_{v} \simeq\left(\bar{K}^{>0}, \cdot, 1,<\right) .
$$

Consequently, $1+I_{v} \subset \mathcal{U}_{v}^{>0}$ is a jump, i.e. there is no convex subgroup $C$ such that $1+I_{v} \varsubsetneqq C \varsubsetneqq \mathcal{U}_{v}^{>0}$.

Proof: Follows immediately from the properties of the residue map together with (5). The last assertion follows from the fact that $\bar{K}$ is archimedean.

By virtue of Lemmas 2.15, 3.7 and 3.6, we now obtain:

Theorem 3.8 If $\left(K^{>0}, \cdot, 1,<\right)$ is divisible, then there exist a group complement $\mathbf{B}$ to $\mathcal{U}_{v}^{>0}$ in $\left(K^{>0}, \cdot, 1,<\right)$ and a group complement $\mathbf{B}^{\prime}$ to $1+I_{v}$ in $\left(\mathcal{U}_{v}^{>0}, \cdot, 1,<\right)$ such that

$$
\left(K^{>0}, \cdot, 1,<\right)=\mathbf{B} \amalg \mathbf{B}^{\prime} \amalg\left(1+I_{v}, \cdot, 1,<\right) .
$$

Every group complement to $\mathcal{U}_{v}^{>0}$ in $\left(K^{>0}, \cdot, 1,<\right)$ is isomorphic to $G$, and every group complement to $1+I_{v}$ in $\left(\mathcal{U}_{v}^{>0}, \cdot, 1,<\right)$ is isomorphic to $\left(\bar{K}^{>0}, \cdot, 1,<\right)$.

In the sequel, we will compute the natural valuation $w$ and the ordered skeleton of $\left(K^{>0}, \cdot, 1,<\right)$ in dependence upon the natural valuation $v_{G}$ and the ordered skeleton $[\Gamma,\{B(\gamma) ; \gamma \in \Gamma\}]$ of $G$ on the one hand, and upon the natural valuation $v$ and the ordered skeleton of $(K,+, 0,<)$ on the other hand. Although the first part of the preceding theorem requires the divisibility of the multiplicative group of positive elements of $K$, we may use Lemmas 3.6 and 3.7 to compute its skeleton even if divisibility does not hold, by means of Lemma 2.16:

Corollary 3.9 For every ordered field $K$,

$$
S\left(K^{>0}, \cdot, 1,<\right) \simeq S(G) \amalg S\left(\bar{K}^{>0}, \cdot, 1,<\right) \amalg S\left(1+I_{\nu}, \cdot, 1,<\right) .
$$


The value set in $S\left(\bar{K}^{>0}, \cdot, 1,<\right)$ consists of just one element (apart from $\infty$ ) since $\left(\bar{K}^{>0}, \cdot, 1,<\right)$ is archimedean. Consequently, the only component in this skeleton (apart from $\{0\})$ is itself isomorphic to $\left(\bar{K}^{>0}, \cdot, 1,<\right)$.

Our goal is now to give more detailed information on the valuation and the components of $\left(K^{>0}, \cdot, 1,<\right)$. For this, we need some notations. As we are working with three different ordered Abelian groups, for "the smallest convex subgroup containing $x$ " and "the biggest convex subgroup not containing $x$ ", we will write

$$
\begin{aligned}
& C_{x} \text { and } D_{x} \text { for } x \in(K,+, 0,<) \\
& \mathbf{C}_{x} \text { and } \mathbf{D}_{x} \text { for } x \in\left(K^{>0}, \cdot, 1,<\right) \\
& \mathcal{C}_{x} \text { and } \mathcal{D}_{x} \text { for } x \in G .
\end{aligned}
$$

In $\left(K^{>0}, \cdot, 1,<\right)$, for $\sim, \gg$, « we will write $\dot{\sim}, \gg$ and $\ll$ respectively.

Now we are able to extract some more information from Lemmas 3.6 and 3.7, again by means of Lemma 2.16. Note that for every $a \in K^{\times}$, we have $v_{G}(-v(a))=$ $v_{G}(v(a))$.

\section{Lemma 3.10}

a) Suppose that $a, b \in K^{>0}$ and $v(a) \neq 0$. Then $b \ll a$ if and only if $v_{G}(v(b))>$ $v_{G}(v(a))$, and

$$
\mathbf{C}_{a} / \mathbf{D}_{a} \simeq \mathcal{C}_{v(a)} / \mathcal{D}_{v(a)}
$$

b) Suppose that $a, b \in \mathcal{U}_{v}^{>0} \backslash 1+I_{v}$. Then $a \dot{\sim} b$ and

$$
\mathrm{C}_{a} / \mathbf{D}_{a} \simeq\left(\bar{K}^{>0}, \cdot, 1,<\right)
$$

since the latter group is archimedean.

Now it remains to consider $\left(1+I_{v}, \cdot, 1,<\right)$. We will relate its natural valuation to the natural valuation $v$ of the additive group of $K$, and we will show that its skeleton is isomorphic to the skeleton of $\left(I_{v},+, 0,<\right)$.

Lemma 3.11 Suppose that $a>1$ and $b>1$. If $v(a-1)=v(b-1)$, then $a \dot{\sim} b$.

Proof: We set $\varepsilon_{1}=a-1$ and $\varepsilon_{2}=b-1$. By hypothesis, there exists $n>1$ such that $n \varepsilon_{1}>\varepsilon_{2}$ and $n \varepsilon_{2}>\varepsilon_{1}$. We write

$$
\left(1+\varepsilon_{1}\right)^{n}=1+n \varepsilon_{1}+\sum_{i=2}^{n}\left(\begin{array}{c}
n \\
i
\end{array}\right) \varepsilon_{1}^{i} .
$$

But

$$
\varepsilon_{1}>0 \Rightarrow \sum_{i=2}^{n}\left(\begin{array}{c}
n \\
i
\end{array}\right) \varepsilon_{1}^{i}>0
$$

hence

$$
\left(1+\varepsilon_{1}\right)^{n}>1+n \varepsilon_{1}>1+\varepsilon_{2} .
$$

Similarly, one shows that $\left(1+\varepsilon_{2}\right)^{n}>1+\varepsilon_{1}$. 
Lemma 3.12 Suppose that $a>1$ and $b>1$. Let $v(b-1) \geq 0$. If $v(a-1)<v(b-1)$, then $a \gg b$.

Proof: We set $\varepsilon_{1}=a-1$ and $\varepsilon_{2}=b-1$. We want to show for every $n>0$ : $\left(1+\varepsilon_{2}\right)^{n}<1+\varepsilon_{1}$. We have that for every $i>0$,

$$
v\left(\varepsilon_{2}^{i}\right)=i v\left(\varepsilon_{2}\right) \geq v\left(\varepsilon_{2}\right)>v\left(\varepsilon_{1}\right)
$$

and thus,

$$
v\left(\left(\begin{array}{c}
n \\
i
\end{array}\right) \varepsilon_{2}^{i}\right)>v\left(\varepsilon_{1}\right)
$$

whence

$$
v\left(\sum_{i=1}^{n}\left(\begin{array}{c}
n \\
i
\end{array}\right) \varepsilon_{2}^{i}\right)>v\left(\varepsilon_{1}\right) .
$$

In particular (by (5)),

$$
\sum_{i=1}^{n}\left(\begin{array}{c}
n \\
i
\end{array}\right) \varepsilon_{2}^{i}<\varepsilon_{1} .
$$

On the other hand,

$$
\left(1+\varepsilon_{2}\right)^{n}=1+\sum_{i=1}^{n}\left(\begin{array}{c}
n \\
i
\end{array}\right) \varepsilon_{2}^{i},
$$

hence,

$$
\left(1+\varepsilon_{2}\right)^{n}<1+\varepsilon_{1}
$$

The following corollary will serve us to describe the natural valuation of $1+I_{v}$ :

Corollary 3.13 If $a>1, b>1$ and $v(b-1) \geq 0$, then

$$
a \gg b \text { if and only if } v(a-1)<v(b-1) \text {. }
$$

Consequently, the map

$$
a \mapsto v(a-1)
$$

is (equivalent to) the natural valuation on $\left(1+I_{v}, \cdot, 1,<\right)$.

Proof: The first assertion is an immediate consequence of Lemmas 3.11 and 3.12 . The second assertion is a consequence of the first, by virtue of Remark 3.5 iii).

Lemma 3.14 For every $a \in 1+I_{v}$, the assignment $c \cdot \mathbf{D}_{a} \mapsto c-1+D_{a-1}$ establishes an isomorphism

$$
\mathbf{C}_{a} / \mathbf{D}_{a} \simeq\left(C_{a-1} / D_{a-1},+, 0,<\right)
$$

of ordered groups. 
Proof: Assume that $a \in 1+I_{v}$. We define

$$
\begin{aligned}
\phi_{a}: \mathbf{C}_{a} & \longrightarrow C_{a-1} / D_{a-1} \\
c & \mapsto c-1+D_{a-1} .
\end{aligned}
$$

Let us remark that by definition of $\mathbf{C}_{a}$ and Corollary 3.13, $c \in \mathbf{C}_{a}$ if and only if $v(c-1) \geq v(a-1)$. Hence, $\phi_{a}$ is well defined and surjective. Similarly, $c \in \mathbf{D}_{a}$ if and only if $v(c-1)>v(a-1)$. Hence, $\operatorname{Ker} \phi_{a}=\mathbf{D}_{a}$.

It remains to show that $\phi_{a}$ is a homomorphism. Given $c, d \in \mathbf{C}_{a}$, we have to prove that $\phi_{a}(c d)=\phi_{a}(c)+\phi_{a}(d)$, i.e. that

$$
(c d-1)-(c-1)-(d-1) \in D_{a-1} .
$$

This is equivalent to:

$$
v((c-1)(d-1))>v(a-1) .
$$

But this is true since

$$
v((c-1)(d-1))=v(c-1)+v(d-1) \geq 2 v(a-1)>v(a-1) .
$$

Finally, $\phi_{a}$ preserves the order: if $c>1$, then $c-1>0$.

From Corollary 3.13 and Lemma 3.14, we can deduce the following results:

Theorem 3.15 For every ordered field $K$,

$$
S\left(\left(1+I_{\nu}, \cdot, 1,<\right)\right) \simeq S\left(\left(I_{\nu},+, 0,<\right)\right)
$$

Proof: For $v$ the natural valuation on $I_{v}$, Corollary 3.13 shows that $w$ defined by $w(a)=v(a-1)$ is (equivalent to ) the natural valuation on $1+I_{v}$. The component of $1+I_{v}$ corresponding to $\gamma=w(a), a \in 1+I_{v}$, is $\mathbf{C}_{a} / \mathbf{D}_{a}$ (this follows from Lemma 2.16 in view of the convexity of $\left.1+I_{v}\right)$. But by Proposition 3.14, $\mathbf{C}_{a} / \mathbf{D}_{a}$ is isomorphic to $C_{a-1} / D_{a-1}$ which is the component of $I_{v}$ corresponding to $v(a-1)=w(a)$. This proves that the skeletons are isomorphic.

Corollary 3.16 Let $K$ be an ordered field, root closed for positive elements. Then $I_{v} \equiv_{\infty} 1+I_{v}$ as ordered groups. If moreover $K$ is countable, then $K$ admits an isomorphism $f: I_{v} \rightarrow 1+I_{v}$ of ordered groups, that is, $K$ admits a right exponential.

Proof: $\quad$ Since $K$ is root closed for positive elements, $1+I_{v}$ is a $\mathbb{Q}$-vector space. On the other hand, by Corollary 3.15, it has the same skeleton as $I_{v}$. So our assertions follow from part a) of Theorem 2.27 .

For the notions of pseudo Cauchy sequence and pseudo limit appearing in the next lemma, cf. [GRA1]. Here, $\nu, \mu, \rho$ will denote ordinals and $\lambda$ a limit ordinal. 
Lemma 3.17 Let $K$ be any ordered field. A sequence $\left\{a_{\nu} ; \nu<\lambda\right\}$ is pseudo Cauchy in $\left(1+I_{\nu}, \cdot, 1,<\right)$ if and only if $\left\{a_{\nu}-1 ; \nu<\lambda\right\}$ is pseudo Cauchy in $\left(I_{\nu},+, 0,<\right)$. Moreover, $a \in 1+I_{\nu}$ is a pseudo limit of $\left\{a_{\nu} ; \nu<\lambda\right\}$ if and only if $a-1 \in I_{\nu}$ is a pseudo limit of $\left\{a_{\nu}-1 ; \nu<\lambda\right\}$.

Proof: $\quad$ Let $\rho<\mu<\nu<\lambda$. Then by Corollary 3.13,

$$
w\left(\frac{a_{\nu}}{a_{\mu}}\right)>w\left(\frac{a_{\mu}}{a_{\rho}}\right) \text { if and only if } v\left(\frac{a_{\nu}}{a_{\mu}}-1\right)>v\left(\frac{a_{\mu}}{a_{\rho}}-1\right) .
$$

The latter is equivalent to

$$
v\left(\frac{a_{\nu}-a_{\mu}}{a_{\mu}}\right)>v\left(\frac{a_{\mu}-a_{\rho}}{a_{\rho}}\right) .
$$

Since $v\left(a_{\mu}\right)=v\left(a_{\rho}\right)=0$, this in turn is equivalent to

$$
v\left(a_{\nu}-a_{\mu}\right)>v\left(a_{\mu}-a_{\rho}\right)
$$

that is,

$$
v\left(\left(a_{\nu}-1\right)-\left(a_{\mu}-1\right)\right)>v\left(\left(a_{\mu}-1\right)-\left(a_{\rho}-1\right)\right) .
$$

This proves the first assertion. The second assertion is proved similarly.

A valued vector space is maximally valued if and only if every pseudo Cauchy sequence has a pseudo limit (cf. [GRA1]). For the case of valued fields, this was originally shown by Kaplansky [KAP]. For the notion of a power series field appearing in the next corollary, and a proof of the fact that such a field is maximally valued, see [RIB]. Here, let $E$ denote any archimedean ordered field and $G$ any ordered Abelian group.

Corollary 3.18 Every power series field $E((G))$ admits a right exponential.

Proof: Since $E((G))$ is maximally valued, its additive group is maximally valued and consequently, so is $\left(I_{v},+, 0,<\right)$ (by Lemma 2.17). Hence, it follows from the foregoing lemma that $\left(1+I_{v}, \cdot, 1,<\right)$ is maximally valued as well. As a power series field, $E((G))$ is henselian with respect to $v$. By general valuation theory, this implies that its group $1+I_{v}$ of 1-units is divisible. By Theorem 3.15 and Corollary 2.24 it now follows that the ordered groups $I_{v}$ and $1+I_{v}$ must be isomorphic.

Note that this corollary can also be deduced in a different way using a result of B. H. Neumann (cf. [N]), as observed by Alling in [ALL], section 3, pp. 709-710.

The main theorem of this section now follows immediately from Corollary 3.13 , Lemma 3.14 and Lemma 3.2:

Theorem 3.19 Define a valuation

$$
w: K^{>0} \longrightarrow \Gamma^{-}+G^{\geq 0}+\{\infty\}
$$


on the ordered group $\left(K^{>0}, \cdot, 1,<\right)$ as follows: $w(1)=\infty$ and for all $a \neq 1$,

$$
w(a)=\left\{\begin{array}{cl}
v_{G}(v(a)) & \text { if } a \notin \mathcal{U}_{v}^{>0} \\
0 & \text { if } a \in \mathcal{U}_{v}^{>0} \backslash\left(1+I_{v}\right) \\
v(a-1) & \text { if } a \in\left(1+I_{v}\right) .
\end{array}\right.
$$

Then $w$ is (equivalent to) the natural valuation on $K^{>0}$, i.e.

$$
\forall a, b \in K^{>0}: a \ll b \Longleftrightarrow w(a)>w(b) .
$$

For $A(\delta)$ the component of $\left(K^{>0}, \cdot, 1,<\right)$ corresponding to $\delta \in w\left(K^{>0}\right)^{-}$, we have

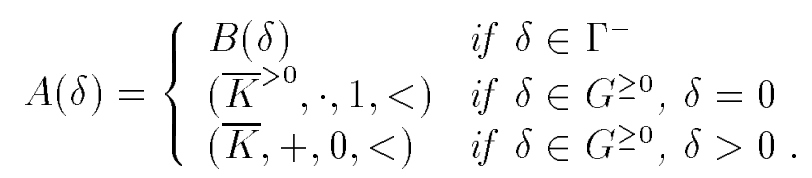

\subsection{Exponential fields}

Let $(K,+, \cdot, 0,1,<)$ be an ordered field. We will say that $K$ is an exponential field if there exists

$$
f:(K,+, 0,<) \longrightarrow\left(K^{>0}, \cdot, 1,<\right)
$$

such that

1) $f$ is an isomorphism of ordered groups

2) $v(f(1)-1)=0$.

A map $f$ with these properties will be called an exponential on $K$. Note that for the definition of "exponential field", condition 2) is in fact superfluous, because if $(K,+, \cdot, 0,1,<)$ admits a map $e$ satisfying 1$)$, then it admits also a map $f$ satisfying 1) and 2): let $a \in K^{>0}$ such that $e(a)=2$, and put $f(x)=e(a x)$. This procedure also shows a possible way how to cope with the fact that axiom 2) is not an elementary axiom if we do not add a symbol for the valuation to our language. Indeed, we are free to replace axiom 2) by the stronger but elementary axiom " $f(1)=2$ ". In the place of 2 , we could choose any other rational number $>1$. As well, we could take two rational numbers $r_{2}>r_{1}>1$ and use the axiom " $r_{1}<f(1)<r_{2}$ ". Finally, by a scheme of axioms of this sort, $f(1)$ may be fixed to any real number, up to addition of an infinitesimal (an element $\varepsilon$ of value $v(\varepsilon)>0$ ).

Lemma 3.20 Let

$$
f:(K,+, 0,<) \rightarrow\left(K^{>0}, \cdot, 1,<\right)
$$

be an isomorphism of ordered groups. Then the following are equivalent:

1) $v(f(1)-1)=0$ (i.e. $f$ is an exponential),

2) the map

$$
\begin{aligned}
\bar{f}:(\bar{K},+, 0,<) & \rightarrow\left(\bar{K}^{>0}, \cdot, 1,<\right) \\
\bar{a} & \mapsto \overline{f(a)}
\end{aligned}
$$

defines an exponential on $\bar{K}$,

3) $f\left(R_{v}\right)=\mathcal{U}_{v}^{>0}$ and $f\left(I_{v}\right)=1+I_{v}$. 
Proof: We first show 1) $\Rightarrow 2$ ).

Assume 1) holds. By Lemma 3.2, the archimedean component corresponding to 1 in $(K,+, 0,<)$ is $(\bar{K},+, 0,<)=R_{v} / I_{v}$, and by part b) of Lemma 3.10 , that corresponding to $f(1)$ in $\left(K^{>0}, \cdot, 1,<\right)$ is $\left(\mathcal{U}_{v}^{>0} / 1+I_{v}, \cdot, 1,<\right)$.

So by Lemma 2.2, the map

$$
\begin{aligned}
& f_{0}: \bar{K} \rightarrow \mathcal{U}_{v}^{>0} / 1+I_{v} \\
& a+I_{v} \mapsto f(a) \cdot\left(1+I_{v}\right)
\end{aligned}
$$

is an isomorphism of ordered groups. Now note that

$$
\mathcal{U}_{v}^{>0} / 1+I_{v}=\left(R_{v} / I_{v}\right)^{>0}=\bar{K}^{>0}
$$

in fact,

$$
\forall b \in \mathcal{U}_{v}^{>0}: b \cdot\left(1+I_{v}\right)=b+b I_{v}=b+I_{v}
$$

since $v(b)=0$ for all $b \in \mathcal{U}_{v}^{>0}$.

So indeed we have that

$$
f_{0}(\bar{a})=\overline{f(a)} .
$$

Assume now that 2 ) holds. It is immediate to see from the properties of $\bar{f}$ that 3 ) must hold.

If 3) holds, then for every $a \in R_{v}$ we have $v(f(a))=0$ and thus $v(f(a)-1) \geq 0$. So 3) implies

$$
\forall a \in R_{v} \backslash I_{v}: v(f(a)-1)=0 .
$$

Since $1 \in R_{v} \backslash I_{v}$, we see that 1 ) holds as required.

Remark 3.21 Suppose that $f$ is an exponential on $K$. Then the multiplicative group of $K$ is divisible since it is isomorphic to the additive group of $K$. Hence, there is a decomposition (8), according to Theorem 3.8. From Lemma 3.20 we know that $f\left(R_{v}\right)=\mathcal{U}_{v}^{>0}$. Hence, $\mathbf{A}=f^{-1}(\mathbf{B})$ is a group complement to $R_{v}$ in $(K,+, 0,<)$. Again from Lemma 3.20 we know that $f\left(I_{v}\right)=1+I_{v}$. Hence, $\mathbf{A}^{\prime}=f^{-1}\left(\mathbf{B}^{\prime}\right)$ is a group complement to $I_{v}$ in $R_{v}$. With these groups $\mathbf{A}$ and $\mathbf{A}^{\prime}$, we have a decomposition (7). Denoting the restriction of $f$ to $\mathbf{A}$ by $f_{L}$, the restriction to $\mathbf{A}^{\prime}$ by $f_{M}$ and the restriction to $I_{v}$ by $f_{R}$, we have obtained isomorphisms

$$
\begin{aligned}
f_{L}: \mathbf{A} & \longrightarrow \mathbf{B} \\
f_{M}: \mathbf{A}^{\prime} & \longrightarrow \mathbf{B}^{\prime} \\
f_{R}: I_{v} & \longrightarrow 1+I_{v} .
\end{aligned}
$$

In view of the display of the lexicographic sums, this motivates the following definition, for every ordered field $K$ with decompositions $(7)$ and (8).

An isomorphism $f_{L}$ from a group complement $\mathbf{A}$ to $R_{v}$ in $(K,+, 0,<)$ onto a group complement $\mathbf{B}$ to $\mathcal{U}_{v}^{>0}$ in $\left(K^{>0}, \cdot, 1,<\right)$ will be called a left exponential; in view of the uniqueness of the group complements, it automatically induces an isomorphism between $\mathbf{A}$ and $G$. Conversely, every isomorphism between $\mathbf{A}$ and $G$ induces a left exponential. 
An isomorphism $f_{M}$ from a group complement $\mathbf{A}^{\prime}$ to $I_{v}$ in $R_{v}$ onto a group complement $\mathbf{B}^{\prime}$ to $1+I_{v}$ in $\mathcal{U}_{v}^{>0}$ will be called a middle exponential; in view of the uniqueness of the group complements, it automatically induces an isomorphism between $(\bar{K},+, 0,<)$ and $\left(\bar{K}^{>0}, \cdot, 1,<\right)$, i.e., an exponential on $\bar{K}$. Conversely, every exponential $e$ on $\bar{K}$ induces a middle exponential $e^{\prime}: \mathbf{A}^{\prime} \simeq \mathbf{B}^{\prime}$.

An isomorphism $f_{R}$ from $I_{v}$ onto $1+I_{v}$ will be called a right exponential.

Given a left exponential $f_{L}$, a middle exponential $f_{M}$ and a right exponential $f_{R}$, then $f_{L} \amalg f_{M} \amalg f_{R}$ is an exponential on $K$, where

$$
\forall a \in \mathbf{A} \forall a^{\prime} \in \mathbf{A}^{\prime} \forall \varepsilon \in I_{v}: f_{L} \amalg f_{M} \amalg f_{R}\left(a+a^{\prime}+\varepsilon\right):=f_{L}(a) \cdot f_{M}\left(a^{\prime}\right) \cdot f_{R}(\varepsilon)
$$

In particular, if $e$ is an exponential on $\bar{K}$ and $e^{\prime}$ the corresponding middle exponential, then $f=f_{L} \amalg e^{\prime} \amalg f_{R}$ is an exponential satisfying $\bar{f}=e$, that is, $f$ lifts $e$.

Every left exponential (and thus also every exponential) $f$ induces an isomorphism

$$
\varphi_{f}: G^{<0} \rightarrow \Gamma^{-}
$$

given by $\varphi_{f}=f_{\mathrm{rk}} \uparrow_{G<0}$. more precisely, $\varphi_{f}$ is defined by

$$
\varphi_{f}(v(a))=w(f(a))=v_{G}(v(f(a))) .
$$

This may be indicated by writing $\varphi_{f}=w \circ f \circ v^{-1}$. Indeed, by Lemma 3.4, the group $\mathbf{A}$ has $G^{<0} \cup\{\infty\}$ as its value set, and the isomorphism $f: \mathbf{A} \rightarrow G$ thus induces the above isomorphism of the value sets. More precisely, $f$ even induces an isomorphism of the skeletons of $\mathbf{A}$ and $G$, so in view of Lemma 3.2, all components of $G$ will be isomorphic to $(\bar{K},+, 0,<)$.

Every right exponential (and thus also every exponential) $f$ induces an automorphism

$$
\psi_{f}: G^{>0} \rightarrow G^{>0}
$$

given by $\psi_{f}=f_{\mathrm{rk}} \uparrow_{G>0}$. It may be indicated by writing $\psi_{f}=w \circ f \circ v^{-1}$ which is defined by $\psi_{f}(v(\varepsilon))=w(f(\varepsilon))=v(f(\varepsilon)-1)$. This is because $G^{>0} \cup\{\infty\}$ is the value set of both $I_{v}$ and $1+I_{v}$. Again, $f$ induces also an isomorphism of the skeletons (but independently of the existence of $f$, we have already shown that the skeletons of $I_{v}$ and $1+I_{v}$ are isomorphic, cf. Corollary 3.15).

As a corollary to Lemma 3.20 and the preceding remark, we obtain the following result which is due to Alling (cf. [ALL], Th. 1.2 and Cor. 1.4).

Corollary 3.22 If $K$ admits an exponential $f$, then

1) $G^{<0} \simeq \Gamma^{-}$as chains,

2) $B(\gamma) \simeq(\bar{K},+, 0,<)$ for every $\gamma \in \Gamma^{-}$,

3) the map

$$
\begin{aligned}
\bar{f}:(\bar{K},+, 0,<) & \longrightarrow \frac{\left(\bar{K}^{>0}, \cdot, 1,<\right)}{\bar{a}} \\
& \mapsto \frac{f(a)}{}
\end{aligned}
$$

is an exponential of $\bar{K}$. 
Note that 1 ) and 2) of this corollary already hold under the assumption that $K$ admits a left exponential.

This last corollary motivates the following definition: Let $G$ be an ordered Abelian group with skeleton $[\Gamma,\{B(\gamma) ; \gamma \in \Gamma\}]$, and denote by $v_{G}$ the natural valuation on $G$ (so $\left.v_{G}(G \backslash\{0\})=\Gamma^{-}\right)$. Further, let $A$ be an archimedean ordered Abelian group. Then $G$ will be called an exponential group in $A$ if

1) it admits a group exponential $\varphi$, i.e. an isomorphism $\varphi: G^{<0} \rightarrow \Gamma^{-}$of chains,

2) $B(\gamma) \simeq A$, for all $\gamma \in \Gamma^{-}$.

With this definition, the statement of the preceding corollary reads as follows:

Theorem 3.23 If $(K, f)$ is an exponential field, then $\left(G, \varphi_{f}\right)$ is a divisible exponential group in $(\bar{K},+, 0,<)$, and $(\bar{K}, \bar{f})$ is an exponential field.

Again, note that the first assertion of this theorem already holds under the assumption that $f$ is a left exponential on $K$.

Another consequence of the above remark is the fact that there are only very special extensions of an exponential field which are immediate with respect to the natural valuations. Let $(L, v) \mid(K, v)$ be an extension of valued (not necessarily exponential) fields. Then $(K, v)$ is said to be dense in $(L, v)$ if for every $a \in L$ and $\alpha \in v\left(L^{\times}\right)$there is some $b \in K$ such that $v(a-b) \geq \alpha$. We first need the following characterization of density. Let $\mathbf{A}_{K}$ be a group complement for the valuation ring of $(K, v)$. Using Zorn's Lemma, it may be extended to a group complement $\mathbf{A}_{L}$ for the valuation ring of $(L, v)$.

Lemma $3.24(K, v)$ is dense in $(L, v)$ if and only if $\mathbf{A}_{K}=\mathbf{A}_{L}$. If this is the case, then $v\left(K^{\times}\right)=v\left(L^{\times}\right)$and $\bar{K}=\bar{L}$, that is, the extension is immediate.

Proof: If $(K, v)$ is dense in $(L, v)$ then for every $a \in L$ with $v(a)<0$ there is some $b \in K$ such that $v(a-b) \geq 0$ which implies that $\mathbf{A}_{K}$ is already a group complement for the valuation ring of $(L, v)$.

For the converse, assume that the latter holds. Let $a \in L$ and $\alpha \in v\left(L^{\times}\right)$. Then $\alpha \in v\left(K^{\times}\right)$since otherwise, an element $d \in L$ with value $v(d)=-|\alpha|$ could not be represented as the sum of an element of $K$ and an element of the valuation ring of $L$. So we may choose some $c \in K$ with $v(c)=\alpha$. By hypothesis, for $a c^{-1}$ there exists some $b^{\prime} \in \mathbf{A}_{K}$ such that $v\left(a c^{-1}-b^{\prime}\right) \geq 0$. Putting $b=b^{\prime} c$, we find $v(a-b) \geq v(c)=\alpha$. This proves the converse.

If $\alpha$ is chosen $>v(a)$, then $v(a-b) \geq \alpha>v(a)$ implies $v(a)=v(b) \in v(K)$ and if $v(a)=0$, also $\bar{a}=\bar{b} \in \bar{K}$. Hence, if $(K, v)$ is dense in $(L, v)$, then we have $v\left(K^{\times}\right)=v\left(L^{\times}\right)$and $\bar{K}=\bar{L}$.

Theorem 3.25 Suppose that $(K, f) \subset(L, f)$ is a nontrivial extension of nonarchimedean exponential fields. Let $v$ denote the natural valuation on both ordered fields and $\Gamma_{L}, \Gamma_{K}$ denote the ranks of $v\left(L^{\times}\right)$resp. $v\left(K^{\times}\right)$. Then either $(K, v)$ is dense in $(L, v)$, or $\Gamma_{L} \backslash \Gamma_{K}$ is infinite.

Proof: Let $\mathbf{A}_{K} \subset \mathbf{A}_{L}$ as above. If $\mathbf{A}_{K}=\mathbf{A}_{L}$, then the foregoing lemma shows that $(K, v)$ is dense in $(L, v)$. Now assume that $\mathbf{A}_{K} \subset \mathbf{A}_{L}$ is a proper extension. 
Composing the exponential $f$ with the canonical epimorphism given in Lemma 3.6 we then find that also $v\left(K^{\times}\right) \subset v\left(L^{\times}\right)$is a proper extension and thus, $v\left(L^{\times}\right) \backslash v\left(K^{\times}\right)$ is infinite. Through the isomorphism $\varphi_{f}$ it follows that $\Gamma_{L} \backslash \Gamma_{K}$ is infinite.

We have seen in Corollary 3.18 that every power series field which is root closed for positive elements, admits a right exponential. As a further consequence of the above remark, let us state here a necessary and sufficient condition for such a field to admit a left exponential:

Proposition 3.26 Let $E((G))$ be a power series field, root closed for positive elements. If $E((G))$ admits a left exponential, then $G$ is maximally valued; more precisely,

$$
G \simeq \underset{G<0}{\mathbf{H}}(E,+, 0,<)
$$

Consequently, $G$ does not admit a valuation basis. Conversely, if $G$ is exponential in $E$ and maximally valued, then $E((G))$ admits a left exponential.

Proof: $\Rightarrow$ : Since $E((G))$ is a power series field, it is maximally valued. That is, the additive group of $E((G))$ with its natural valuation is maximally valued. Assuming the decomposition (7), it then follows by Lemma 2.17 that $\mathbf{A}$ is maximally valued. If $E((G))$ admits a left exponential, then $G$ is isomorphic to $\mathbf{A}$ and thus also maximally valued. Hence, $G$ will be a Hahn product over its skeleton, according to Corollary 2.8. Since $G$ is an exponential group in $(E,+, 0,<)$ by Theorem 3.23 , (11) holds. On the other hand, $G^{<0} \simeq \Gamma^{-}$implies that $\Gamma^{-}$contains infinite well ordered subsets, and by Lemma 2.9 it follows that $G$ does not admit a valuation basis.

$\Leftarrow$ : If $G$ is exponential in $E$ and maximally valued, then $G$ is of the form (11), hence $G \simeq \mathbf{A}$, and this induces a left exponential (cf. Remark 3.21).

Corollary 3.27 Let $E((G))$ be a power series field, root closed for positive elements. Let $e$ be an exponential on $E$. Then $E((G))$ admits an exponential lifting $e$ if and only if $G$ is exponential in $E$ and maximally valued.

Proof: $\quad \Rightarrow$ : follows from Proposition 3.26.

$\Leftarrow$ : By Proposition 3.26, $E((G))$ admits a left exponential. On the other hand, by Corollary 3.18 , it admits a right exponential. The assertion now follows from Remark 3.21.

To prove our next theorem, we need Lemma 3.29 below, which is based on the following result (cf. Fuchs [FU] for a proof):

Lemma 3.28 Let $\varphi: H \rightarrow J$ be an isomorphism of ordered subgroups of $\mathbb{R}$. Then there exists an element $r \in \mathbb{R}^{>0}$ such that $\varphi(a)=r \cdot a$ for every $a \in H$.

Lemma 3.29 Let $H, J$ be archimedean ordered groups. If $H \equiv_{\infty} J$ (as ordered groups), then $H \simeq J$ (as ordered groups). 
Proof: We may suppose that $H$ and $J$ are subgroups of $\mathbb{R}$ (cf. Theorem 3.1 ). Let $\varphi_{0}: H_{0} \rightarrow J_{0}$ be a local isomorphism of a subgroup $H_{0} \subset H$ onto a subgroup $J_{0}$ of $J$. Then there exists $r \in \mathbb{R}^{>0}$ such that $\varphi_{0}\left(a_{0}\right)=r \cdot a_{0}$ for every $a_{0} \in H_{0}$. Hence, we have that $r \cdot H_{0} \subset J$. Let us show that $r \cdot H \subset J$. Let $a \in H$ and $H_{1}$ be the subgroup of $H$ generated by $H_{0} \cup\{a\}$, and

$$
\varphi_{1}: H_{1} \longrightarrow J_{1}
$$

a local isomorphism such that $\varphi_{0} \subset \varphi_{1}$. Then there exists $s \in \mathbb{R}^{>0}$ such that $\varphi_{1}\left(a_{1}\right)=s \cdot a_{1}$ for every $a_{1} \in H_{1}$. It follows that $r=s$ and hence, $r \cdot a \in J$.

By a symmetrical argument, considering

$$
\begin{aligned}
\varphi_{0}^{-1}: J_{0} & \longrightarrow H_{0} \\
b_{0} & \mapsto r^{-1} \cdot b_{0},
\end{aligned}
$$

it may be shown that $r^{-1} \cdot J \subset H$, i.e. $J \subset r \cdot H$. It follows that $J=r \cdot H$ and hence,

$$
\begin{aligned}
\varphi: H & \longrightarrow J \\
a & \mapsto r \cdot a
\end{aligned}
$$

is the required isomorphism.

\section{Remark 3.30}

There are two essential ingredients for the proof of our next theorem:

i) We will use Theorem 2.27. In fact, $(K,+, 0,<)$ and $\left(K^{>0}, \cdot, 1,<\right)$ being divisible ordered Abelian groups, we consider them as ordered $\mathbb{Q}$-vector spaces. It is important to verify here the following general fact. Let $G_{1}, G_{2}$ be divisible ordered Abelian groups; then $G_{1} \equiv_{\infty} G_{2}$ as ordered groups if and only if $G_{1} \equiv_{\infty} G_{2}$ as ordered $\mathbb{Q}$-vector spaces. Let us briefly sketch a proof of the nontrivial direction. Let $F: G_{1} \simeq_{p} G_{2}$ as ordered groups; it suffices to find $\tilde{F}: G_{1} \simeq_{p} G_{2}$ such that for every $\tilde{f} \in \tilde{F}$, $\operatorname{dom} f$ and $\operatorname{im} f$ are divisible ordered Abelian groups (every isomorphism of divisible ordered Abelian groups being an isomorphism of ordered $\mathbb{Q}$-vector spaces). The construction of $\tilde{F}$ from $F$ is done without difficulty as follows. If $f \in F, \operatorname{dom} f=H_{1}, \operatorname{im} f=H_{2}$, and if $\tilde{H}_{1}$ (resp. $\tilde{H}_{2}$ ) denote the divisible closures of $H_{1}\left(\operatorname{resp} . H_{2}\right)$ in $G_{1}\left(\operatorname{resp} . G_{2}\right)$, then $f$ extends in a unique way to an isomorphism

$$
\tilde{f}: \tilde{H}_{1} \longrightarrow \tilde{H}_{2}
$$

and we take

$$
\tilde{F}=\{\tilde{f} ; f \in F\}
$$

ii) Every dense linear ordering without endpoints is $\aleph_{0}$-saturated. Indeed, the theory of dense linear orderings without endpoints is complete and admits elimination of quantifiers (cf. $[\mathrm{R}-\mathrm{Z}])$. Consequently, realizing a 1-type over a finite set 
of parameters reduces to solving finitely many inequalities of the form $x<a$ and $x>b$. But this is always possible in such an ordering.

It follows by Theorem 2.26 that any such ordering is $L_{\infty}$-equivalent to $\mathbb{Q}$. Conversely, if a chain is $L_{\infty}$-equivalent to $\mathbb{Q}$, then it is necessarily a dense linear ordering without endpoints.

We will further use the notations of Theorem 3.19.

Theorem 3.31 Let $K$ be a nonarchimedean ordered field such that $\left(K^{>0}, \cdot, 1,<\right)$ is divisible. Then $(K,+, 0,<) \equiv_{\infty}\left(K^{>0}, \cdot, 1,<\right)$ as ordered groups if and only if

1) $G \equiv_{\infty} \amalg_{\mathbb{Q}}(\bar{K},+, 0,<)$ as ordered groups,

2) $\bar{K}$ admits an exponential.

Proof: $\quad \Rightarrow$ : By part a) of Theorem 2.27, $S((K,+, 0,<)) \equiv_{\infty} S\left(\left(K^{>0}, \cdot, 1,<\right)\right)$ as ordered skeletons. By Theorem 3.19 and part b) of Theorem 2.27, we obtain that

i) $G \equiv{ }_{\infty} \Gamma^{-}+G^{\geq 0}$ as chains,

ii) for every $\gamma \in \Gamma^{-}, B(\gamma) \equiv_{\infty}(\bar{K},+, 0,<)$ as ordered groups,

iii) $\left(\bar{K}^{>0}, \cdot, 1,<\right) \equiv_{\infty w}(\bar{K},+, 0,<)$ as ordered groups

(since all archimedean components of $(K,+, 0,<)$ are isomorphic to $(\bar{K},+, 0,<)$ ). But $\left(K^{>0}, \cdot, 1,<\right)$ being divisible, $G$ is also divisible and hence as a chain, is a dense linear ordering without endpoints (let us remark that $G \neq 0$ since $K$ is nonarchimedean). By i), it follows that $\Gamma^{-}+G^{\geq 0}$ is a dense linear ordering without endpoints, so the same holds for $\Gamma^{-}$. By part ii) of the preceding remark, it follows that

$$
\Gamma^{-} \equiv{ }_{\infty \omega} \mathbb{Q} .
$$

Let $\Phi: \Gamma^{-} \simeq_{p} \mathbb{Q}$. Since by ii) and Lemma 3.29 all nontrivial archimedean components of $G$ are isomorphic to $(\bar{K},+, 0,<)$, it is easy to construct from $\Phi$ a family

$$
\Phi^{\prime}: S(G) \simeq_{p} S\left(\coprod_{\mathbb{Q}}(\bar{K},+, 0,<) .\right.
$$

So assertion 1) follows now from part a) of Theorem 2.27, and assertion 2) follows from iii) and Lemma 3.29.

$\Leftarrow$ : Let us assume the decompositions (7) and (8) (see Lemma 3.4 and Theorem 3.8). Let $e$ be an exponential on $\bar{K}$ and $e^{\prime}$ the induced middle exponential:

$$
e^{\prime}: \mathbf{A}^{\prime} \simeq \mathbf{B}^{\prime}
$$

By Corollary 3.16, we know that

$$
I_{v} \equiv_{\infty} 1+I_{v}
$$

So our assertion will follow as soon as

$$
\mathbf{A} \equiv_{\infty \omega} \mathbf{B}
$$

is proved. In fact, if $F_{L}: \mathbf{A} \simeq_{p} \mathbf{B}$ and $F_{R}: I_{v} \simeq_{p} 1+I_{v}$, then

$$
F:=F_{L} \amalg\left\{e^{\prime}\right\} \amalg F_{R}:(K,+, 0,<) \simeq_{p}\left(K^{>0}, \cdot, 1,<\right)
$$


where $F_{L} \amalg\left\{e^{\prime}\right\} \amalg F_{R}=\left\{f_{L} \amalg e^{\prime} \amalg f_{R} \mid f_{L} \in F_{L}, f_{R} \in F_{R}\right\}$ (cf. definition (10)). But $v(\mathbf{A})^{-}=G^{<0}$ is a dense linear ordering without endpoints, so

$$
G^{<0} \equiv{ }_{\infty \omega} \mathbb{Q} \text {. }
$$

On the other hand, all components of $\mathbf{A}$ are isomorphic to $(\bar{K},+, 0,<)$, so by an argument already used in the first part of this proof, it follows that

$$
S(\mathbf{A}) \equiv_{\infty \omega} S\left(\coprod_{\mathbb{Q}}(\bar{K},+, 0,<)\right)
$$

It follows by part a) of Theorem 2.27 that

$$
\mathbf{A} \equiv \varlimsup_{\infty} \coprod_{\mathbb{Q}}(\bar{K},+, 0,<)
$$

But $\mathbf{B} \simeq G$, so together with the hypothesis of the theorem we obtain that

$$
\mathbf{B} \equiv \equiv_{\infty} \coprod_{\mathbb{Q}}(\bar{K},+, 0,<) \equiv_{\infty} \mathbf{A}
$$

As a corollary, we obtain the following theorem which gives us the "converse"

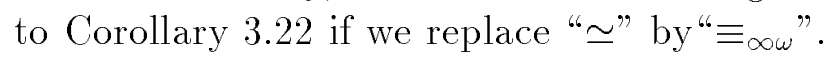

Corollary 3.32 Let $K$ be a nonarchimedean ordered field such that $\left(K^{>0}, \cdot, 1,<\right)$ is divisible. Then $(K,+, 0,<) \equiv_{\infty}\left(K^{>0}, \cdot, 1,<\right)$ as ordered groups if and only if

1) $G^{<0} \equiv{ }_{\infty w} \Gamma^{-}$as chains,

2) $B(\gamma) \simeq(\bar{K},+, 0,<)$ for every $\gamma \in \Gamma^{-}$,

3) $\bar{K}$ admits an exponential.

Proof: By arguments already used in the proof of the previous theorem, one can see that conditions 1 ) and 2) together are equivalent to condition 1) of the theorem.

Note that by part ii) of Remark 3.30, assertion 1) is equivalent to the property of $\Gamma^{-}$to be a dense linear ordering without endpoints. Note also that by virtue of Lemma 3.29, the corollary is true also for archimedean fields.

For the countable case, the above theorem yields:

\section{Theorem 3.33 (Countable Case Characterization Theorem)}

Let $K$ be a countable nonarchimedean ordered field such that $\left(K^{>0}, \cdot, 1,<\right)$ is divisible. Given an exponential e on $\bar{K}$, it can be lifted to an exponential $f$ on $K$ (i.e., $\bar{f}=e)$ if and only if $G \simeq \coprod_{\mathbb{Q}}(\bar{K},+, 0,<)$.

Proof: $\Rightarrow$ follows from Theorem 3.31 and the fact that $G$ and $\bar{K}$ are countable, by virtue of Theorem 2.25 .

$\Leftarrow$ : Given an exponential $e$ on $\bar{K}$, let $\epsilon^{\prime}$ and $F$ be as in the proof of Theorem 3.31. Then every element $f_{0}$ of $F$ extends $e^{\prime}$. So by virtue of Theorem 2.25, there exists 
an isomorphism $f:(K,+, 0,<) \rightarrow\left(K^{>0}, \cdot, 1,<\right)$ extending $e^{\prime}$. It follows that $\bar{f}=e$.

Note that the condition on $G$ in the above theorem is actually equivalent to the assertion " $G$ is an exponential group in $(\bar{K},+, 0,<)$ "; in fact, we have the following

Proposition 3.34 Let $G \neq 0$ and $A$ be countable divisible ordered Abelian groups and let $A$ be archimedean. Then $G$ is an exponential group in $A$ if and only if it is of the form $G \simeq \coprod_{\mathbb{Q}} A$.

Proof: $\Rightarrow$ : Let $G$ be a countable exponential group in $A$. Then $G^{<0} \simeq \Gamma^{-}$, so $\Gamma^{-}$is a countable dense linear ordering without endpoints. By a classical theorem of Cantor, it follows that $\Gamma^{-} \simeq \mathbb{Q}$ as chains. On the other hand, all components of $G$ are isomorphic to $A$, so by part a) of Theorem 2.27 , we have $G \simeq \coprod_{\mathbb{Q}} A$.

$\Leftarrow$ : Let $G \simeq \coprod_{\mathbb{Q}} A$. Then $\Gamma^{-} \simeq \mathbb{Q}$, and $G^{<0}$ is a countable dense linear ordering without endpoints. Again by Cantor's Theorem, $G^{<0} \simeq \Gamma^{-}$. Since all components of $G$ are $A$, it follows that $G$ is exponential in $A$.

From the proof of Theorem 3.31 we obtain the following corollary which contains the symmetrical analogue to Corollary 3.16 :

Corollary 3.35 Let $K$ be an ordered field, root closed for positive elements.

a) If $G$ is an exponential group in $(\bar{K},+, 0,<)$, then $G \equiv_{\infty} \amalg_{\mathbb{Q}}(\bar{K},+, 0,<)$.

b) Assume the decompositions (7) and (8). Then $\mathbf{A} \equiv_{\infty} \mathbf{B}$ (as ordered groups) if and only if $G \equiv_{\infty} \coprod_{\mathbb{Q}}(\bar{K},+, 0,<)$. If moreover $K$ is countable, then $K$ admits an isomorphism $f: \mathbf{A} \rightarrow \mathbf{B}$ of ordered groups (that is, $K$ admits a left exponential) if and only if $G \simeq \coprod_{\mathbb{Q}}(\bar{K},+, 0,<)$.

Remark 3.36 In all the preceding theorems and corollaries, we may replace the function "exponential" by " $\bar{K}-$ linear exponential". Indeed, the appearing value groups are not only $\mathbb{Q}$ - but also $\bar{K}$-vector spaces, and Theorem 2.27 holds for arbitrary ordered vector spaces. This is of interest because also the usual exponential on $\mathbb{R}$ is in fact $\mathbb{R}$-linear.

To finish this paper, let us say a word about the hypothesis of divisibility for $\left(K^{>0}, \cdot, 1,<\right)$. In fact, Theorem 2.27 used in the proof of Theorem 3.31 is not true if one omits the hypothesis of divisibility. The following example, due to F.-V. Kuhlmann, shows that there exist two regular countable ordered Abelian groups, having isomorphic skeletons, which are not isomorphic as ordered groups. (An ordered Abelian group $A$ is regular if and only if $A / B$ is divisible, for every nontrivial convex subgroup $B$ of $A$.)

Example 3.37 In $\mathbb{Q} \oplus \mathbb{Q}$, take a subgroup $H$ such that the projection to the first component is $\mathbb{Q}$ and the projection to the second component is $\frac{1}{2^{\infty}} \mathbb{Z}$, but which is not a direct sum of $\mathbb{Q}$ and $\frac{1}{2^{\infty}} \mathbb{Z}$ (the existence of such a group is well known). On $\mathbb{Q} \oplus \mathbb{Q}$, take the lexicographic order, and on $H$ the restriction of this order. We have

$$
H / C(H,(0,1)) \simeq \mathbb{Q}
$$


which shows the regularity of $H$. The archimedean components are $\mathbb{Q}$ and $\frac{1}{2^{\infty}} \mathbb{Z}$, but $H \neq \mathbb{Q} \oplus \frac{1}{2^{\infty}} \mathbb{Z}$ implies that

$$
H \nsucc \mathbb{Q} \amalg \frac{1}{2^{\infty}} \mathbb{Z}
$$

\section{References}

[ALL] Alling, N. L.: On exponentially closed fields, Proc. A. M. S. 13 No. 5 (1962), 706-711

[BAR] Barwise, J. (ed.): Back and forth through infinitary logic, in: Studies in Model Theory, edited by M. Morley, Math. Assoc. Am., Buffalo, NY, 5-34

[BR] Brown, R.: Valued vector spaces of countable dimension, Publ. Math. Debrecen 18 (1971), 149-151

[C-K] Chang, C. C. - Keisler, H. J.: Model Theory, Amsterdam - London (1973)

[D-L] van den Dries, L. - Lewenberg, A.: T-convexity and tame extensions, to appear in the J.S. L.

[D-M-M] van den Dries, L. - Macintyre, A. - Marker, D. : The elementary theory of restricted analytic functions with exponentiation, preprint

[D-W] Dahn, B. I. - Wolter, H.: On the theory of exponential fields, Zeitschr. f. math. Logik und Grundlagen d. Math. 29 (1983), 465-480

[FU] Fuchs, L.: Partially ordered algebraic systems, Addison-Wesley, Reading, Mass., (1963)

[GRA1] Gravett, K. A. H.: Valued linear spaces, Quart. J. Math. Oxford (2), $6(1955), 309-315$

[GRA2] Gravett, K. A. H.: Ordered Abelian groups, Quart. J. Math. Oxford (2), 7 (1956), 57-63

[KAP] Kaplansky, I.: Maximal fields with valuations I, Duke Math. Journ. 9 (1942), 303-321

[KF1] Kuhlmann, F.-V.: Valuation Theory of Fields, Abelian Groups and Modules, to appear in the "Algebra, Logic and Applications" series, eds. Gbel and Macintyre, by Gordon and Breach.

[KF2] Kuhlmann, F.-V.: Abelian groups with contractions I, in: Proceedings of the Oberwolfach Conference on Abelian Groups 1993, AMS Contemporary Mathematics 171 (1994) 
[KF3] Kuhlmann, F.-V.: Abelian groups with contractions II: weak o-minimality, erscheint in Proceedings of the Padua Conference on Abelian Groups 1994

[K-K1] Kuhlmann, F.-V.- Kuhlmann, S.: On the structure of nonarchimedean exponential fields II, Comm. in Algebra 22(12) (1994), 5079-5103

[K1] Kuhlmann, S.: Quelques propriétés des espaces vectoriels valués en théorie des modèles, Thesis Paris (1991)

[K2] Kuhlmann, S.: $L_{\infty}$-equivalence of valued and ordered vector spaces, in preparation

[M-W] Macintyre, A..J.-Wilkie, A.J. : On the decidability of the real exponential field: Preprint

[N] Neumann, B. H.: On ordered division rings, T. A. M. S. 66 (1949), $202-252$

[POI] Poizat, B.: Cours de théorie des modèles, Villeurbanne (1985)

[RE] Ressayre, J.-P.: Integer parts of real closed exponential fields, preprint

[RIB] Ribenboim, P.: Théorie des valuations, Les Presses de l'Université de Montréal, 2nd edition, Montréal (1968)

[R-Z] Robinson, A. - Zakon, E.: Elementary properties of ordered abelian groups, T. A. M. S. 96 (1960), 222-236

[W] Wilkie, A. J.: Model completeness results for expansions of the real field, to appear in the American Journal of Mathematics

Mathematisches Institut

Universität Heidelberg

Im Neuenheimer Feld 288

D-69120 Heidelberg, Germany 